\title{
Propagation of a solitary wave over a finite submerged thin plate
}

DOI:

10.1016/j.apor.2020.102425

\section{Document Version}

Accepted author manuscript

Link to publication record in Manchester Research Explorer

\section{Citation for published version (APA):}

Christou, A., Stoesser, T., Xie, Z., \& Ouro, P. (2021). Propagation of a solitary wave over a finite submerged thin plate. Applied Ocean Research, 106, [102425]. https://doi.org/10.1016/j.apor.2020.102425

\section{Published in:}

Applied Ocean Research

\section{Citing this paper}

Please note that where the full-text provided on Manchester Research Explorer is the Author Accepted Manuscript or Proof version this may differ from the final Published version. If citing, it is advised that you check and use the publisher's definitive version.

\section{General rights}

Copyright and moral rights for the publications made accessible in the Research Explorer are retained by the authors and/or other copyright owners and it is a condition of accessing publications that users recognise and abide by the legal requirements associated with these rights.

\section{Takedown policy}

If you believe that this document breaches copyright please refer to the University of Manchester's Takedown Procedures [http://man.ac.uk/04Y6Bo] or contact uml.scholarlycommunications@manchester.ac.uk providing relevant details, so we can investigate your claim.

\section{OPEN ACCESS}




\title{
Propagation of a solitary wave over a finite submerged thin plate
}

\author{
Aristos Christou $^{\mathrm{a}}$, Zhihua Xie ${ }^{\mathrm{b}}$, Thorsten Stoesser ${ }^{\mathrm{a}, *}$, Pablo Ouro $^{\mathrm{b}}$ \\ ${ }^{a}$ Department of Civil, Environmental and Geomatic Engineering, University College London, Gower Street, WC1E 6BT, \\ London, UK \\ ${ }^{b}$ School of Engineering, Cardiff University, Cardiff, CF24 3AA, UK
}

\begin{abstract}
For the purpose of this paper, the in-house large-eddy simulation code, Hydro3D, is refined to study wave structure interaction. First of all, the code is used to develop a numerical wave tank capable of simulating accurately the generation, progression and damping of solitary waves in a tank. Then, Hydro3d is employed to simulate a previous laboratory experiment of a wave propagating over an infinitely wide flat plate. The code's accuracy is validated by comparing computed waterlevels and hydrodynamic forces on the plate with measured data for which good agreement is found for a number of conditions (i.e. varying wave steepness or plate submergence, respectively). Then the study is extended to investigate three-dimensional effects for which the infinitely wide plate is replaced by a finite square plate. It is found that the pressure difference between the lower and upper side of the plate drives a span-wise flow and creates unique flow structures and water-surface fluctuations near the plate due to the three-dimensionality of the problem. A further three-dimensional study is conducted for which the finite plate is fixed at an angle of attack in respect to the incident wave and variations in hydrodynamic forces and free-surface elevations are computed. Both vertical and horizontal forces are reduced when the plate is fixed at $45^{\circ}$ degrees and minor water-level fluctuations appear, reflecting the pattern of the rotational flow near the plate edges. Plots of the velocity vectors, swirlstrength, pressure and wave elevation and acting forces reveal significant differences between an infinitely wide and a finite square plate subjected to a solitary wave.
\end{abstract}

Keywords: 3D NWT, LES, Solitary wave, Wave-structure interaction, Turbulent flow

\section{Introduction}

The experience of natural extreme events such as tsunami and storm waves due to global warming and climate change are becoming more frequent phenomena and can have catastrophic consequences for coastal environments. Extreme waves are found to have a major impact on coastal structures since most of them are often designed to operate in relatively calm water conditions. An in-depth understanding and analysis of such structures interacting with extreme waves is necessary to prevent fatal damages and to adopt more safe and cost-effective designs.

\footnotetext{
${ }^{*}$ Corresponding author

Email address: t.stoesser@ucl.ac.uk (Thorsten Stoesser)
} 
Bridges, break-waters and wave energy converters are often found near the coast and parts of their design can be idealised as infinitely wide plates [1]. The simple geometry of a flat plate makes it an ideal benchmark plates have focused on the prediction of wave loads, pressure, flow field and free-surface elevations [2-7].

In a comprehensive review, $\mathrm{Yu}$ [8] lists numerical and experimental methods to analyse the behavior of horizontal fixed plates under periodic and solitary waves. Moreover, the effects of length and submergence of a horizontal plate and how these can lead to an efficient break-water or wave energy converter are investigated. field close to a horizontal plate using particle image velocimetry (PIV) confirming the presence of energetic vortices near the edges of the plate.

Based on velocity potential, results published in Liu et al. [16] obtained from a modified desingularized boundary integral equation method (DBIEM), were validated with the experimental data of Brossard et Ertekin $[4,5]$ used the level 1 Green-Naghdi (GN) equations to evaluate the forces acting on a submerged flat plate by solitary and cnoidal waves. The above methods predict the forces and free-surface elevation near submerged flat plates to a sufficient degree of accuracy. On the other hand, potential flow and the models presented above maybe incapable of accurately capturing complex rotational flows surrounding submerged Dynamics (CFD) and the increase in computing power, several numerical models that solve the Navier-Stokes (N-S) equations to study coastal engineering problems have recently been developed. Detailed reviews on the application of the above can be found in Gotoh et al. [18].

CFD is widely adapted to a very wide range of applications such as in hydraulics $[19,20]$ and coastal (DNS), Reynolds Average Navier-Stokes (RANS) models, Large Eddy Simulations (LES) or a hybrid of the latter two, Detached Eddy Simulations (DES). For example, Xie et al. [21] developed a 3D LES NWT based on the dynamic Smagorinsky sub-grid scale (SGS) model [22] and Volume of Fluid (VOF) method [23] to study among others, solitary waves interacting with flat plates, reproducing the experimental study of Lo and Liu [2] where numerical results of waterlevels, pressures and forces acting on the plate, agreed well with the experimental data. Lo and Liu [2] developed a 2D RANS model coupled with VOF method to capture the fluid structure interaction of submerged flat plates. Pressure forces, moments and free-surface elevations have been calculated and compared with experimental data revealing that their model is able to predict 
with reasonable accuracy these quantities. For instance, a non-zero net moment due to an oscillating force as the wave passes over the plate was observed in the experiment and the numerical predictions agreed well with the observation. Similarly, Ai et al. [6] developed a non-hydrostatic 2D RANS model to solve only the liquid phase (water) and they studied solitary waves travelling over suspended structures. Their numerical results of the free-surface elevation, pressure, forces and moment compared well with experimental data and they extended their investigation by looking at the effect of plate's length and submergence on mentioned quantities for several wave conditions. Alternatively, You et al. [24, 25] studied the interaction of solitary waves with a horizontal flat plate adopting a Constrained Interpolation Profile (CIP) model to solve the 2D N-S equations coupled with a Tangent of Hyperbola for Interface Capturing (THINC) scheme to capture the free-surface. Various wave-heights and plate submergences were considered and numerical results of wave elevations and forces acting on the plate showed good agreement with relevant numerical and experimental studies. Few other studies exist in the literature such as Seiffert et al. [3] and Hayatdavoodi et al. [26] in which the (inviscid) Euler equations coupled with VOF were employed in the form of the open-source CFD software OpenFOAM to simulate the same problem. To the best of authors knowledge, this is the first time a 3D large eddy simulation coupled with level-set method is employed to study solitary waves interacting with a flat plate.

This paper reports on the propagation of a solitary wave over a (more realistic) finite flat plate with the goal to reveal the effects of three-dimensionality of this wave-structure-interaction problem on waterlevels and wave loads on the plate. In addition, the flow is visualised and instantaneous flow structures and resulting pressure variations around the plate are revealed. Therefore, the method of Large Eddy Simulation (LES) rather than a RANS model, coupled with Level Set Method (LSM), is employed. The results presented in this study will support future work and help for better understanding of the local flow hydrodynamics involved in tsunami-like waves interacting with realistic engineering structures. Section 2 describes the methodology and numerical implementations of the current model followed by validations of the model applied in various plate submergence and wave steepness conditions, in Section 3. Results and discussion of a fully three-dimensional wave - finite plate interaction are presented in Section 4. Main conclusion and future work are discussed in Section 5 .

\section{Numerical framework}

In this study the in-house code Hydro3D, validated for many flows of engineering interest [27, 28], is employed for the simulations. Hydro3D solves the unsteady, incompressible, viscous spatially filtered Navier-Stokes equations, written in tensor notation:

$$
\frac{\partial \bar{u}_{i}}{\partial x_{i}}=0
$$




$$
\frac{\partial \bar{u}_{i}}{\partial t}+\frac{\partial \bar{u}_{i} \bar{u}_{j}}{\partial x_{j}}=-\frac{1}{\rho} \frac{\partial \bar{p}}{\partial x_{i}}+\frac{\partial}{\partial x_{j}}\left(\nu \frac{\partial \bar{u}_{i}}{\partial x_{j}}\right)-\frac{\partial \tau_{i j}^{S G S}}{\partial x_{j}}+f_{i}+g_{i}+S_{i}
$$

75 using an iterative multi-grid technique until the intermediate velocity $\tilde{u}_{i}{ }^{*}$ satisfying the continuity equation, Eq. (1), as:

$$
\frac{\partial}{\partial x_{i}}\left(\frac{\partial \tilde{p}}{\partial x_{i}}\right)=\frac{\partial \tilde{u}_{i}^{*}}{\partial x_{i}} \frac{1}{\Delta t}
$$

The current time-step's velocity $u_{i}^{t}$ and pressure $p^{t}$, are then evaluated using:

$$
\begin{gathered}
u_{i}^{t}=\tilde{u}_{i}{ }^{*}-\Delta t \frac{1}{\rho} \frac{\partial \tilde{p}}{\partial x_{i}} \\
p^{t}=p^{t-1}+\tilde{p}-\frac{\nu \Delta t}{2} \frac{\partial}{\partial x_{j}}\left(\frac{\partial \tilde{p}}{\partial x_{j}}\right)
\end{gathered}
$$




\subsection{Free surface capture}

Hydro3D has been refined by Kara et al. [35] to simulate the flow dynamics of submerged bridges, by implementing an interface capturing method capable of predicting complex free-surface deformations which affect significantly the hydrodynamics of the flow [36], [37]. In Hydro3D, the interface of the two phases is captured using the Level Set Method (LSM) based on Osher and Sethian [38]. In LSM a level set, signed signed distance function is defined as follows:

$$
\phi(x, t)\left\{\begin{array}{lll}
<0, & \text { if } & x \in \Omega_{\text {gas }} \\
=0, & \text { if } & x \in \Gamma \\
>0, & \text { if } & x \in \Omega_{\text {liquid }}
\end{array}\right.
$$

where $\Omega_{\text {gas }}$ stands for the volume occupied by air, $\Omega_{\text {liquid }}$ by water and $\Gamma$ is their interface. Since the interface moves with the fluid particles, a pure advection equation is expressed as follows and discretised using a 5th-order weighted essentially non- oscillatory (WENO) scheme [39]:

$$
\frac{\partial \phi}{\partial t}+u_{i} \frac{\partial \phi}{\partial x_{i}}=0
$$

In order to avoid discontinuities in density $(\rho)$ and viscosity $(\mu)$ between the two phases, a transition zone in which density and viscosity are smoothly switched between the air and the water, is employed using the Heaviside function $H(\phi)$ as:

$$
\begin{aligned}
& H(\phi) \begin{cases}=0, & \text { if } \quad \phi<-\varepsilon \\
=\frac{1}{2}\left(1+\frac{\phi}{\varepsilon}+\frac{1}{\pi} \sin \frac{\pi \phi}{\varepsilon}\right), & \text { if } \quad|\phi| \leq \varepsilon \\
=1, & \text { if } \phi>\varepsilon\end{cases} \\
& \rho(\phi)=\rho_{g}+\left(\rho_{l}-\rho_{g}\right) H(\phi) \\
& \mu(\phi)=\mu_{g}+\left(\mu_{l}-\mu_{g}\right) H(\phi)
\end{aligned}
$$

where notations $g$ and $l$ represent gas and fluid variables and $\varepsilon$ is half the thickness of the transition zone. Once the free-surface is obtained, density and dynamic viscosity $(\rho(\phi)$ and $\mu(\phi)$, respectively) are updated at every cell in the computational domain based on Eq. (10) and Eq. (11). In the current study $\varepsilon=2 * \max (\Delta x, \Delta y, \Delta z)$. Finally, to accomplish that $\phi$ maintain its property, $|\nabla \phi|=1$, and avoid nonconserved issues a re-initialization technique introduced by Sussman [40] is adopted as:,

$$
\frac{\partial \phi}{\partial t_{a}}+s\left(\phi_{0}\right)(|\nabla \phi|-1)=0
$$

where $s\left(\phi_{0}\right)$ is the smoothed signed function defined as:

$$
s\left(\phi_{0}\right)=\frac{\phi_{0}}{\sqrt{\phi_{0}^{2}+\left(\left|\nabla \phi_{0}\right| \varepsilon_{r}\right)^{2}}}
$$


where $t_{a}$ is an artificial time calculated based on the grid size multiplied by a factor of less than one. $\phi_{0}(x, 0)=\phi(x, t)$ and $\varepsilon_{r}$ represents one grid size. Equation (12) and Eq. (13) are solved only inside the transition zone in several inner iterations. In Eq. (12), $\phi$ is the signed distance function obtained from the advection equation (Eq. (9)) before applied to re-initialization.

\subsection{Wave generation/absorption}

First of all, Hydro3D is employed to develop a numerical wave tank (NWT). Dirichlet boundary conditions for water surface elevation and the three components of the fluid velocity are prescribed to generate waves at the inlet of the computational domain. These are based on analytical wave theories such as for solitary waves, Stokes waves, cnoidal waves etc. Here, solitary waves are generated using the Boussinesq theory presented in Lee et al. [41]:

$$
\begin{gathered}
n(x, t)=H_{0} \operatorname{sech}^{2}\left(K\left(x-c t-x_{0}\right)\right) \\
\frac{u}{\sqrt{g d}}=\epsilon\left[n_{*}-\frac{1}{4} \epsilon n_{*}^{2}+\left.\frac{d^{2}}{3 c^{2}}\left(1-\frac{3 z^{2}}{2 d^{2}}\right) \frac{\partial^{2} n_{*}}{\partial t^{2}}\right|_{t=0}\right] \\
\frac{w}{\sqrt{g d}}=z \frac{\epsilon}{c}\left[\left(1-\frac{1}{2} \epsilon n_{*}\right) \frac{\partial n_{*}}{\partial t}+\left.\frac{d^{2}}{3 c^{2}}\left(1-\frac{z^{2}}{2 d^{2}}\right) \frac{\partial^{3} n_{*}}{\partial t^{3}}\right|_{t=0}\right]
\end{gathered}
$$

where $n$ is the wave elevation measured from still water-level $(n=0$ at $z=d), H_{0}$ the wave amplitude, $d$ the water-depth, $K=\sqrt{\frac{3 H_{0}}{4 d^{3}}}$ defines the wave-number, wave celerity $c=\sqrt{g\left(H_{0}+d\right)}, n_{*}=\frac{n}{H_{0}}, \epsilon=\frac{H_{0}}{d}$ and $x_{0}$ is the initial position of the wave crest. For a solitary wave, wave-period $\mathrm{T}$ and effective wave-length $\lambda$ can be defined as:

$$
T=\frac{2 \pi}{K c} \quad, \quad \lambda=\frac{2 \pi}{K} .
$$

At the domain's outlet, Hydro3D waves are absorbed using the artificial damping method based on Choi and Yoon [42]. The source term $S_{i}$ generates an artificial damping layer inside which the incident waves are effectively absorbed and reads:

$$
S_{i}=-a_{i}\left(f_{1}+f_{2}\left|u_{i}\right|\right) \Gamma(X) u_{i}
$$

A blending function $\Gamma(X)$ is used to blend the damping term based on a non-dimensional variable $X$ which takes values from 0 , at the boundary of the effective domain and the damping zone, to 1 at the outflow boundary of the domain and is set as:

$$
\Gamma(X)=\frac{e^{X^{R}}-1}{e^{1}-1} \quad, \quad X=\frac{x-x_{s}}{x_{e}-x_{s}}=[0,1]
$$

For the purpose of this study $a_{i}=0$ for $i=1,2$ and $a_{i}=1$ for $i=3$ thus, absorption is only adopted in the vertical direction. Coefficients $f_{1}$ and $f_{2}$ tune the absorbing method and have units of $1 / s$ and $1 / m$, 
respectively. $x_{s}$ and $x_{e}$ stand for the start and end coordinate of the absorption layer in the streamwise direction, respectively and $\mathrm{R}$ describes the blending function (i.e $\mathrm{R}=1$, damping increases almost linearly in the x-direction). In the current study $f_{1}=f_{2}=40, R=2$ and the length of the damping zone is usually twice the wave-length. Peric and Abdel-Maksoud [43] presented a method to scale the damping coefficients according to the generated wave parameters. A solitary wave of different steepness is generated using the above equations and according to Lo and Liu [2] at a water-depth of $0.2 \mathrm{~m}$ and compared well with theoretical results, as shown in Fig. 1. Minor tail waves for high $H_{0} / d$ ratios presented in the numerical results are also common in laboratory experiments.

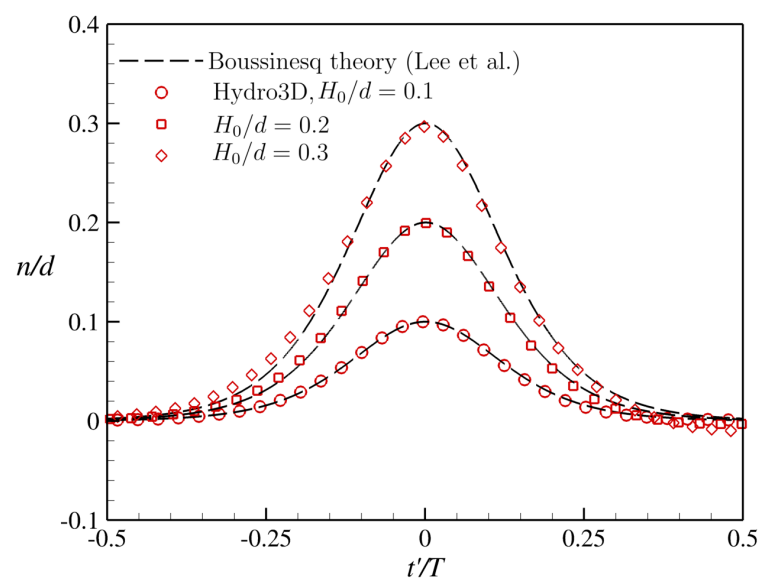

Fig. 1: Normalized wave elevation of simulated solitary waves compared with theory for different $H_{0} / d$ ratios. Similar to Fig.3 in $[2]$

\section{Validation}

In this section, the experimental study of a solitary wave interacting with a fixed horizontal plate conducted by Seiffert et al. [3] is reproduced and their data are used to validate the numerical method. Simulation results published in the same study using OpenFOAM, referred to as 'Seiffert et al.' in the following figures, are also used for comparison. In the laboratory experiment, four different water-depths and several plate submergences and wave-steepnesses are considered and the forces acting on the submerged plate were measured. The following section considers a challenging case with a relatively shallow water-depth and relatively steep breaking waves which serves to validate Hydro3D.

Table 1: Wave-plate parameters

\begin{tabular}{|ccccccc|}
\hline$\alpha(\mathrm{m})$ & $\mathrm{d}(\mathrm{m})$ & $H_{0} / d$ & $d^{\prime} / d$ & $\delta(\mathrm{m})$ & $\mathrm{T}(\mathrm{sec})$ & $\mathrm{L}(\mathrm{m})$ \\
\hline 0.305 & 0.086 & $0.1-0.5$ & $0.2-0.6$ & 0.0127 & $2.21-0.78$ & $2.11-0.88$ \\
\hline
\end{tabular}


Table 2: Exact steepness ratio $H_{0} / d$

\begin{tabular}{c|c}
\hline$H_{0} / d$ referred & $H_{0} / d$ exact \\
\hline 0.1 & 0.087 \\
0.2 & 0.177 \\
0.3 & 0.287 \\
0.4 & 0.396 \\
0.5 & 0.505 \\
\hline
\end{tabular}

3.1. Solitary wave interacting with a horizontal fixed plate.

(a) Top view

\begin{tabular}{|c|c|cc|}
\hline Y WG1 & & WG2 & WG3 \\
\hline
\end{tabular}

(b) Side view

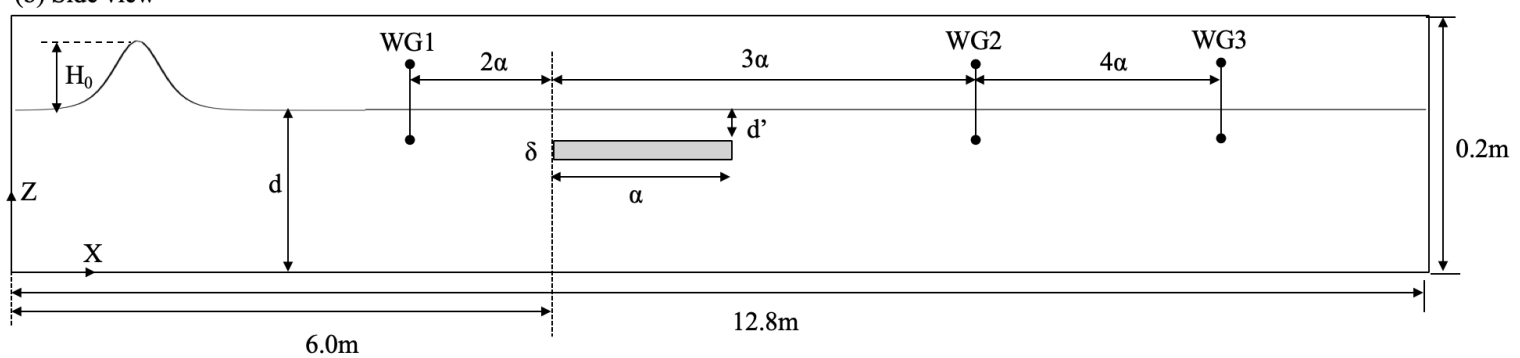

Fig. 2: Schematic diagram of the computational domain showing the plate and locations of the wave probes. (a) Top view, (b) side view. Not to scale.

Figure 2 sketches a solitary wave, normalised amplitude $H_{0} / d$, and the submerged plate of submergence $\left(d^{\prime}\right)$. The water depth is fixed at $d=0.086 \mathrm{~m}$. Three wave-gauges surround the plate of length $\alpha$, that is located approximately half-way along the domain in the streamwise direction. WG1 is located two platelengths upstream of the Leading Edge (LE) of the plate, whereas WG2 and WG3 are placed two and six plate-lengths downstream of the Trailing Edge (TE), respectively. Table 1 lists the relevant geometrical and wave parameters. Five different wave steepnesses, referred to as $H_{0} / d=0.1,0.2,0.3,0.4,0.5$ and two plate submergences $d^{\prime} / d=0.2,0.6\left(d^{\prime}=0\right.$ at $\left.z=d\right)$ are considered. The exact wave steepness, as measured in the laboratory, are used in the NWT at the inlet, and these are given in Table 2.

The computational domain size is $12.8 m(x) \times 0.1 m(y) \times 0.2 m(z)$. This ensures that the incident wave is fully developed before it reaches the plate and no reflected waves from the outlet boundary interact with the plate during the simulated time. Analogue to the laboratory flume the plate extends over the entire width and periodic boundary conditions are applied in the $y$-direction representing an infinitely wide plate. A periodic b.c is used to represent a relatively wide NWT by applying an inflow condition at the south 
boundary according to the simulated kinematics of the north boundary ensuring that mass is conserved over the spanwise direction. A Neumann boundary condition for the pressure is adopted at all boundaries. The top of the domain is treated with a slip condition.

Table 3: Numerical cases for convergence and validation tests.

\begin{tabular}{|lllll|}
\hline case & $N_{x}$ & $N_{y}$ & $N_{z}$ & Total cells \\
\hline case 1 & 2560 & 20 & 80 & $4.4 \times 10^{5}$ \\
case 2 & 5120 & 40 & 80 & $1.6 \times 10^{6}$ \\
case 3 & 5120 & 40 & 160 & $32.7 \times 10^{6}$ \\
case 4 & 5120 & 40 & 240 & $49.2 \times 10^{6}$ \\
\hline
\end{tabular}

To determine spatial discretization, a mesh convergence study is conducted adopting several grid resolutions, details of which are presented in Table 3. Figure 3 shows the average error of the water surface elevation recorded by WG1 and WG2 in all four cases. The average error $\bar{\epsilon}$, is calculated based on the present model's numerical results and the experiment conducted by Seiffert et al. [3]. Experimental data are first applied to a time based interpolation, to share the same temporal discretization as in the simulations and an instant error $\epsilon$ is evaluated based on the following:

$$
\epsilon=\left|\frac{n(t)-n_{\exp }(t)}{n_{\exp }(t)}\right|
$$

The error is then time-averaged to obtain $\bar{\epsilon}$ which is less than $1.5 \%$ in all cases.
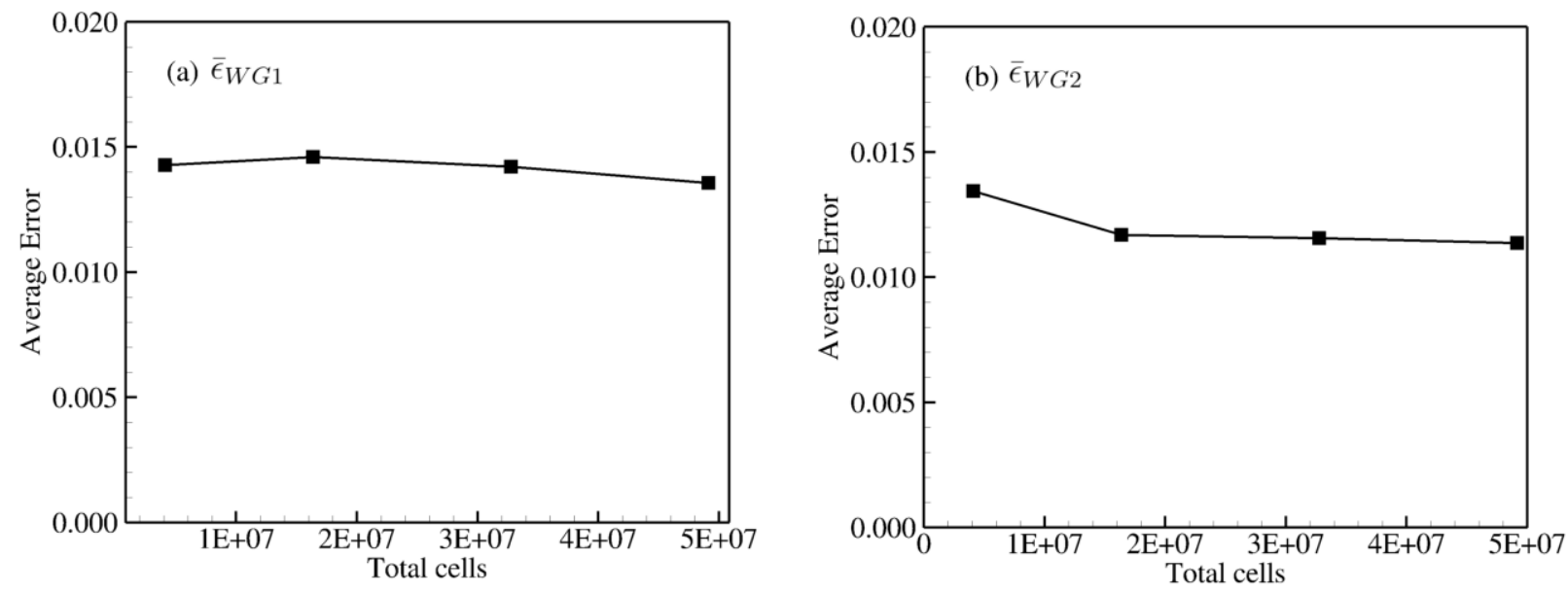

Fig. 3: Average error between numerical results and experimental data at (a) WG1 and (b) WG2 for all four grid resolutions. $d^{\prime} / d=0.2$ and $H_{0} / d=0.3$.

In addition to that, convergence tests are applied to all wave-gauge's locations and forces acting on the plate as shown in Fig. 4 and Fig. 5, respectively. Figures 3 to 5 show that simulation results converge to those obtained on the finest mesh and therefore, the mesh resolution of case 3 is adopted in the current 
study, resulting in a uniform grid resolution of $\Delta x=\Delta y=0.0025 \mathrm{~m}, \Delta z=0.00125 \mathrm{~m}$ (with an aspect ratio, $\mathrm{AR}=2$ ). For temporal discretization, a fixed time-step is used ensuring that the Courant-Friedrichs-Lewy

(i.e. $C F L\left(u_{\max }\right)<1$ ). A fixed time-step of $\Delta t=0.001 \mathrm{~s}$ is adopted for wave-steepnesses of $H_{0} / d=0.1$ to $H_{0} / d=0.3$ whereas for steeper waves, $\Delta t=0.0005 \mathrm{~s}$ is used to accurately capture wave propagation and breaking. The domain is divided into 512 sub-domains and memory is shared between blocks by Message Passing Interface (MPI). For each case of the following section, 512 CPU's are used and the computational time needed to simulate ten seconds of wave propagation is 6 and 12 hours for $\Delta t=0.001 \mathrm{~s}$ and $\Delta t=0.0005 \mathrm{~s}$, respectively.

\subsection{Results and validations.}

Recorded time series of the free-surface, vertical $\left(F_{z}\right)$ and horizontal $\left(F_{x}\right)$ forces of a single case, $d^{\prime} / d=0.2$ and $H_{0} / d=0.3$ are presented in Figs. 4 and 5 , based on a non-dimensional time variable $t^{\prime} / T$ to account for the different plate locations between the current study and the experiments. $t^{\prime}=t-t_{0}$, where $t_{0}$ is the instance at which the peak of the wave reaches WG1. Since pressure at each time-step is known, forces presented below are evaluated by integrating the calculated pressure of each computational cell located at the surface of the plate.

Figure 4 presents calculated water surface elevations (solid lines), experimental data (open squares) and simulation results of Seiffert et al. at the three wave gauges. The computed wave elevations at WG1, WG2 and WG3 agree well with the experimental data. The peak of the incident wave before and after it has passed the plate is well captured by Hydro3D. Moreover, the reflected waves observed after the wave has reached the plate matches well with what was observed in experiment. At WG2 and WG3 Seiffert et al. overestimate the peak of the wave, which is mainly due to the neglecting of viscous effects in their model. Viscous flow inside the boundary layer over the plate dissipates energy due to friction leading to a reduced wave height downstream of the plate. In their study, Seiffert et al. [3] state that the frictional contribution to the total horizontal force $F_{x}$ is small, nevertheless the free-surface appears to be affected quite significantly. 

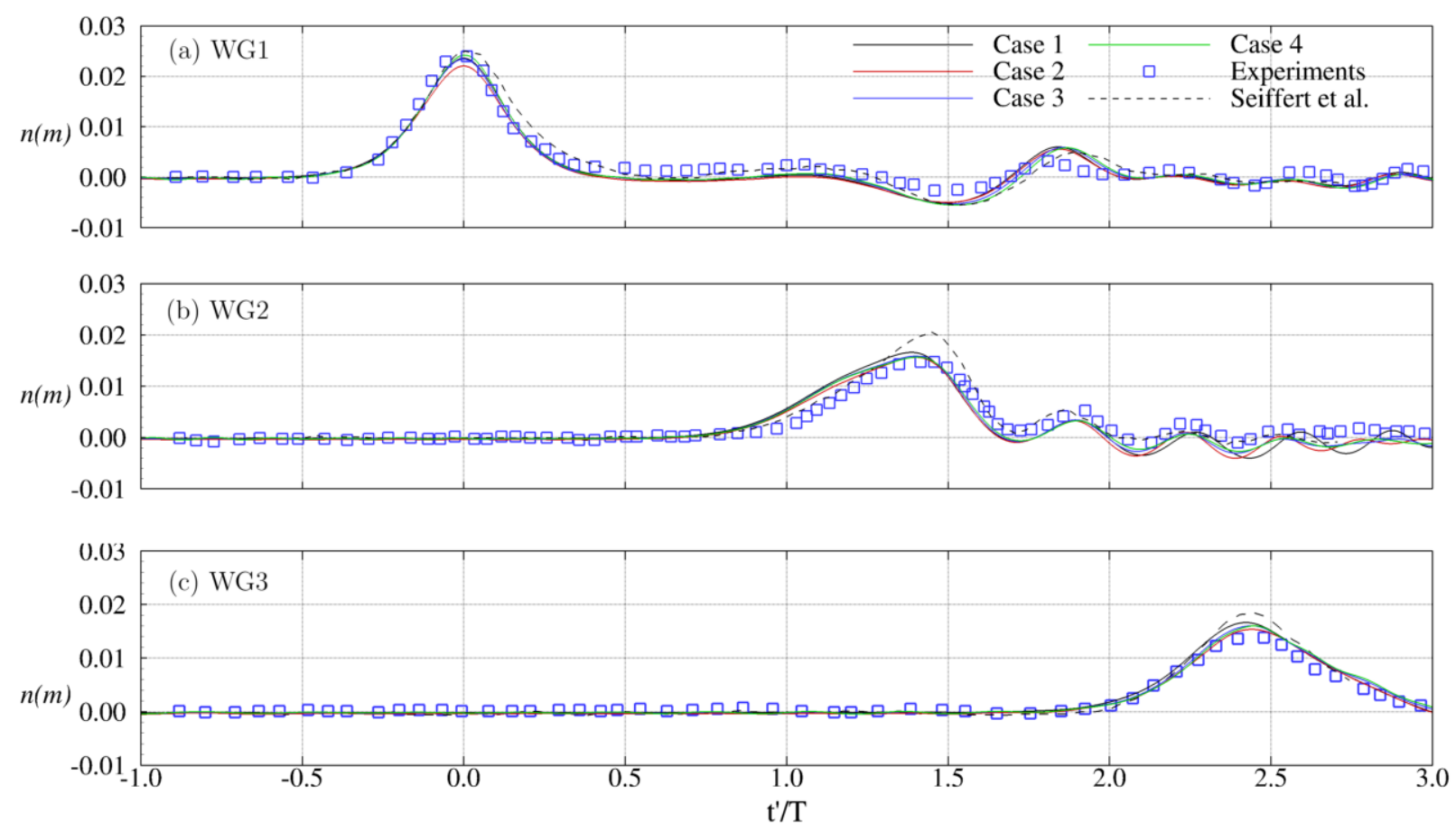

Fig. 4: Calculated free-surface elevations (solid lines), experimental data (open squares) and simulation results of Seiffert et al. at the three wave gauges (a) WG1, (b) WG2, (c) WG3 for $d^{\prime} / d=0.2$ and $H_{0} / d=0.3$.
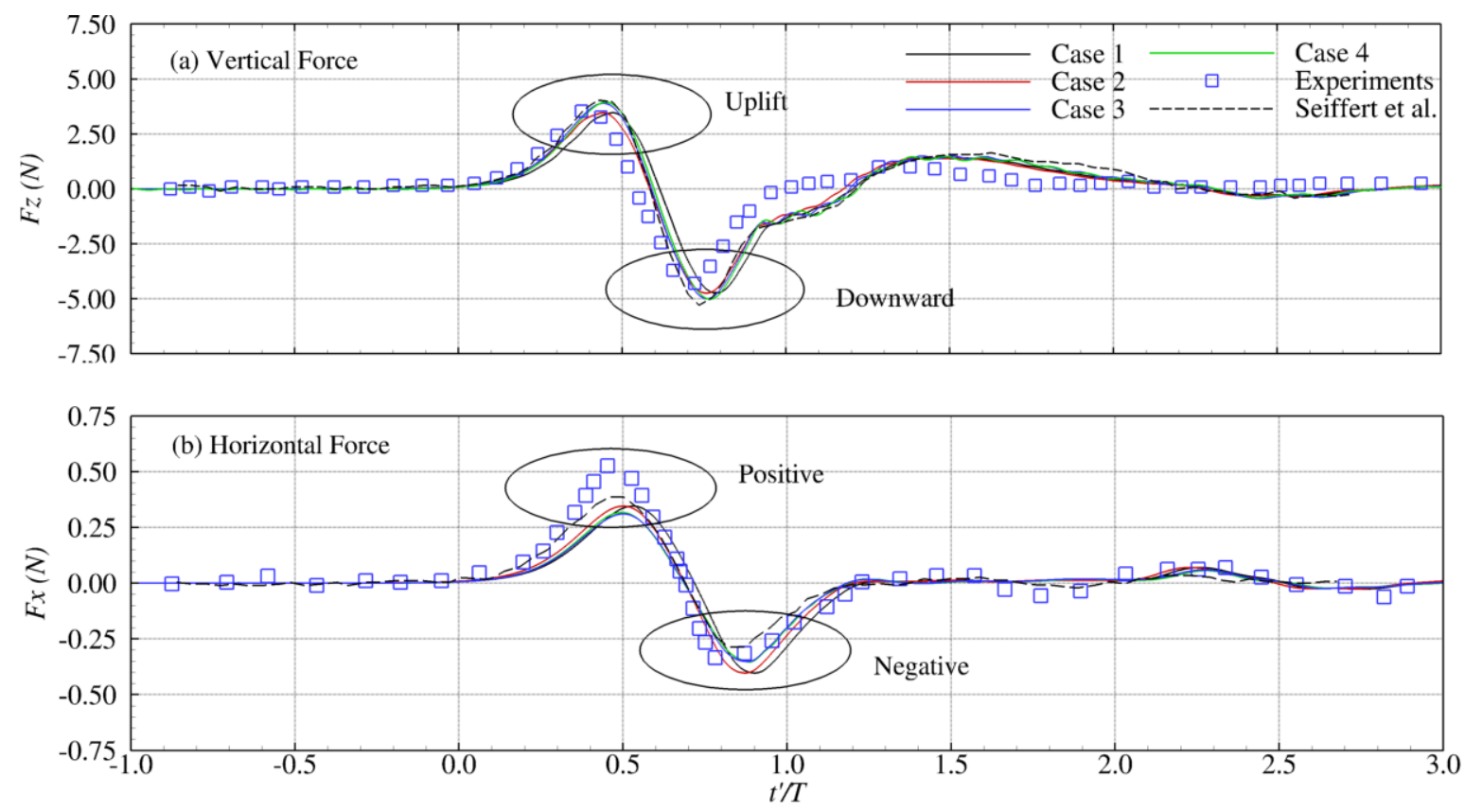

Fig. 5: Calculated plate forces (solid lines), experimental data (open squares) and simulation results of Seiffert et al.: (a) vertical force and (b) horizontal force.

Vertical $\left(F_{z}[N]\right)$ and horizontal $\left(F_{x}[N]\right)$ plate forces as a function of time as calculated by Hydro3D 
(solid lines) are plotted together with experimental data (open squares) and simulation results of Seiffert

et al. in Fig. 5. Hydro3D's predictions are consistent with the numerical results of [3] and agree well with the experimental data for both force components. The calculated positive peak horizontal force is slightly underestimated in all grid resolutions whereas better agreement with the experimental data is observed in the negative peak of the horizontal force. This inconsistency is probably due to the thinness of the plate and only very few computational cells are used to integrate pressure. To confirm this, the calculated viscous force is compared with the pressure horizontal force and results show that the effect of viscosity is considerably small. A similar phenomenon was reported in Seiffert et al. [3] and thus the same approach is used, based on the Blasius' solution, maximum horizontal velocity (from Eq. (15) at $z=d, u_{\max }=0.245 \mathrm{~m} / \mathrm{s}$ ) and Reynolds number $\left(R e_{a}=u_{\max } \alpha / \nu=7.5 \times 10^{4}\right)$ to quantify the viscous drag force, $F_{d}$. By solving Eq. (21), the resultant viscous force is less than $4 \%$ of the maximum calculated horizontal force. In the following equation $C_{f}$ is the frictional drag coefficient and $w$ the width of the plate.

$$
C_{f}=\frac{1.328}{\sqrt{R e_{a}}}=4.85 \times 10^{-3} \quad, \quad F_{d}=2 C_{f}\left(\frac{1}{2} \rho u_{\max }^{2} \alpha\right) w=0.0132 N
$$
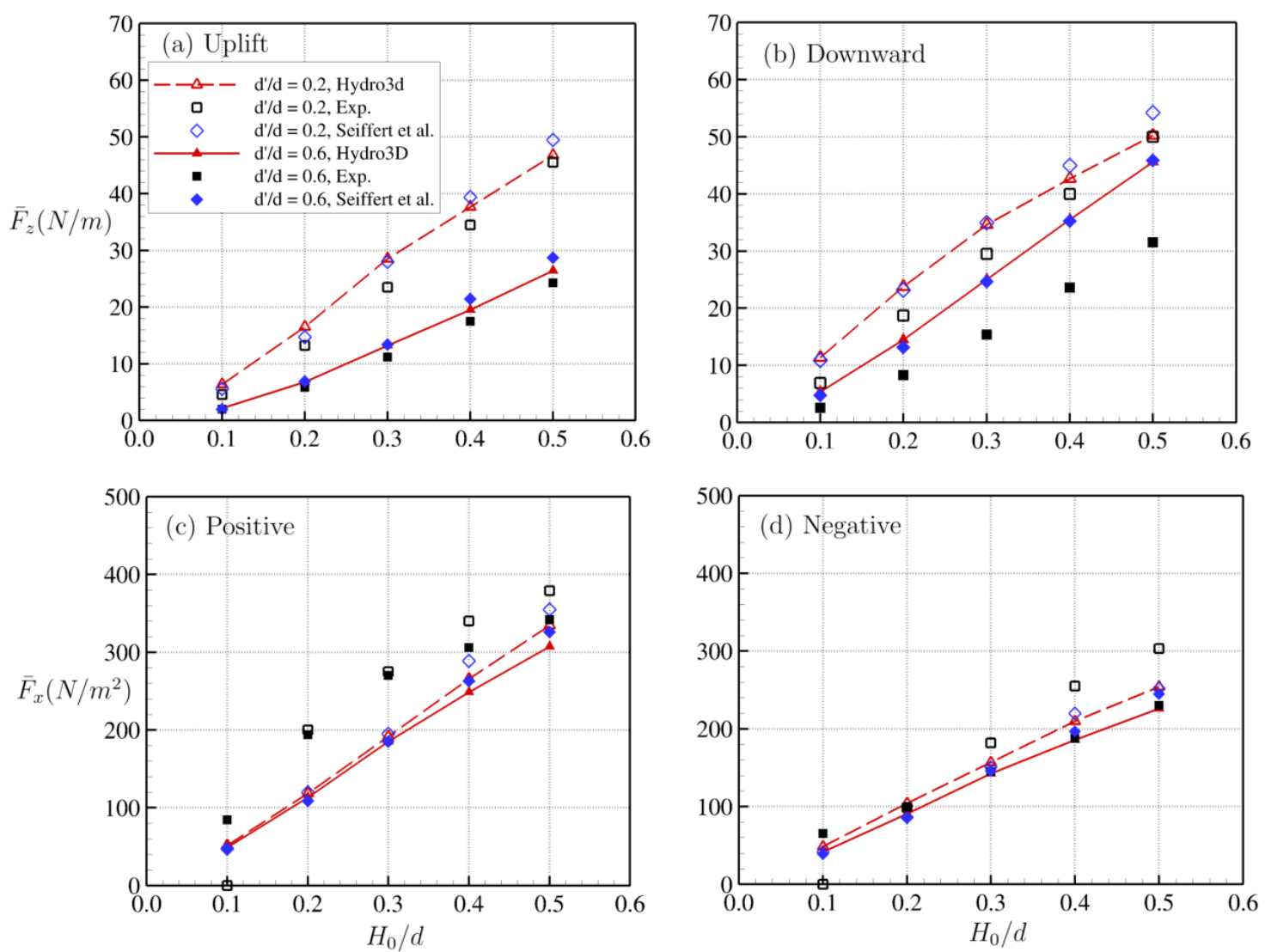

Fig. 6: Peaks of vertical and horizontal forces as predicted by Hydro3d and Seiffert et al. and experimental data. (a) peak uplift force, (b) peak downward force, (c) peak positive force, and (d) peak negative force. 
The peaks of the vertical force per unit width (inside the page) $\left(\bar{F}_{z}[N / m]\right)$ and horizontal force per unit width per unit plate thickness $(\delta)\left(\bar{F}_{x}\left[N / m^{2}\right]\right)$ of ten cases, are presented in Fig. 6. Uplift and downward vertical forces increase with increasing wave steepness and lower plate submergence. In addition, plate submergence appears to have a more significant impact on the uplift force for steeper waves than wave amplitude. On the other hand, horizontal forces are not affected by the plate's submergence as much as vertical forces. Peak horizontal forces increase with increasing wave steepness. Overall, the resultant peak forces computed by Hydro3D for all ten cases agree quite well with the numerical [3] and experimental data, especially in the cases where breaking waves occur. However variations with the experimental data are observed mainly due to the thinness of the plate and few cells are used to integrate pressure. Furthermore, the support of the plate to an aluminum strut and the calculation of forces in the experiments may also induce these discrepancies with the simulations.

Figure 7 plots the space and time evolution of the solitary-wave as it travels along the computational domain. When the wave reaches the plate's LE (plate's centre-line is indicated by the dashed line at $x=6.1525 \mathrm{~m}$ ) the wave height increases due to the decrease of the effective water-depth and initial wave reflections generated from the interaction of the wave with the LE are observed, traveling upstream of the submerged plate. The wave then breaks at approximately $0.25 a$ downstream of the trailing edge (at $x=6.4$ m) and minor free-surface oscillations are generated. Considering the wave-height of the incident wave at WG1 and the maximum elevation recorded by WG2 (Fig. 4a and Fig. 4b, respectively) the wave is reduced by $30 \%$ in height due to energy dissipation in wave-breaking.

In summary, the results presented in this section agree well with the relevant experimental data and hence Hydro3D is considered validated for such wave-structure interaction problems.

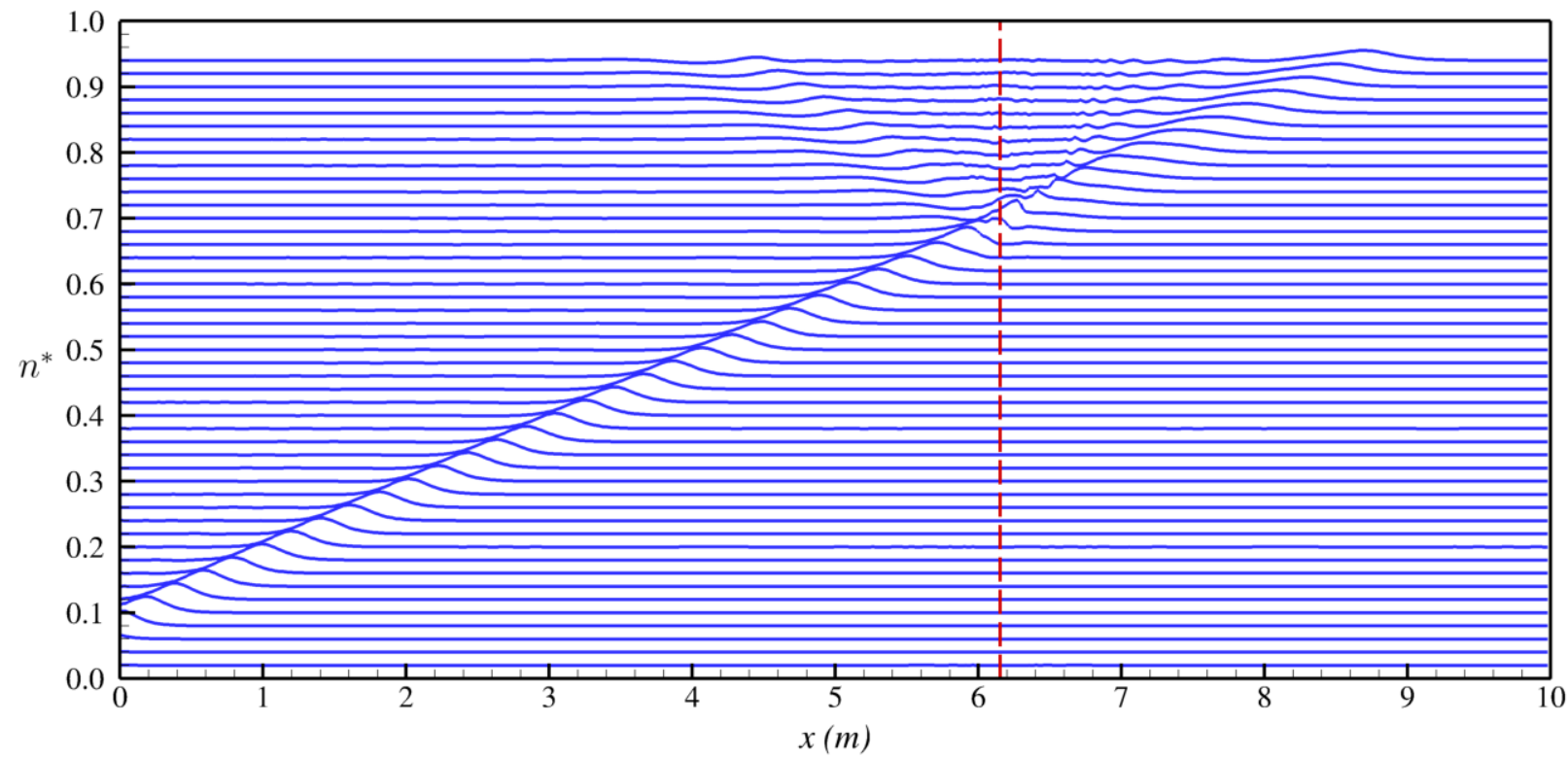

Fig. 7: Computed free-surface elevation in time and space, $n^{*}=n+i * 0.02, i$ is the time-step and the dashed line shows the centre-line of the plate. $d^{\prime} / d=0.2, H_{0} / d=0.3$. 


\section{Wave-plate interaction}

Following the case of an infinitely wide plate in Section 3, a more realistic finite plate is studied which is to be considered a fully three-dimensional, wave-plate interaction problem. A square, submerged plate is placed in the 3D NWT as shown in Fig. 8a. Plots and figures of the simulated free-surface elevation, vortices and local flow hydrodynamics are presented and discussed.

\subsection{Computational setup}

The present study is an extension of one of the cases presented in Section 3.2 with water-depth of $d=0.086 \mathrm{~m}$, wave amplitude of $H_{0} / d=0.3$ and plate submergence of $d^{\prime} / d=0.2$. Table 4 lists the wave and plate parameters used for this particular case. The width of the domain is extended to $2.0 \mathrm{~m}(\sim 6.5 \alpha)$ ensuring that the plate is far away from the side walls to avoid reflections. The length and height of the flume are kept to $12.8 \mathrm{~m}(11 L)$ and $0.2 \mathrm{~m}(2.3 d)$, respectively. The plate is placed midway in the spanwise direction of the domain, and three wave-lengths away from the wave-maker with $0.305 \mathrm{~m}$ in length, $0.305 \mathrm{~m}$ in width and $0.0127 \mathrm{~m}$ thickness, see Fig. 8b. Nine wave-gauges are located in the numerical wave tank to capture the free-surface elevation. The exact position of WG1-WG9 are shown in Fig. 8b.

Table 4: Wave and plate parameters of the 3D case.

\begin{tabular}{|cccccccc|}
\hline$\alpha(\mathrm{m})$ & $\mathrm{d}(\mathrm{m})$ & $H_{0} / d$ & $H_{0} / d$ exact & $d^{\prime} / d$ & $\delta(\mathrm{m})$ & $\mathrm{T}(\mathrm{sec})$ & $\mathrm{L}(\mathrm{m})$ \\
\hline 0.305 & 0.086 & 0.3 & 0.287 & 0.2 & 0.0127 & 1.117 & 1.164 \\
\hline
\end{tabular}

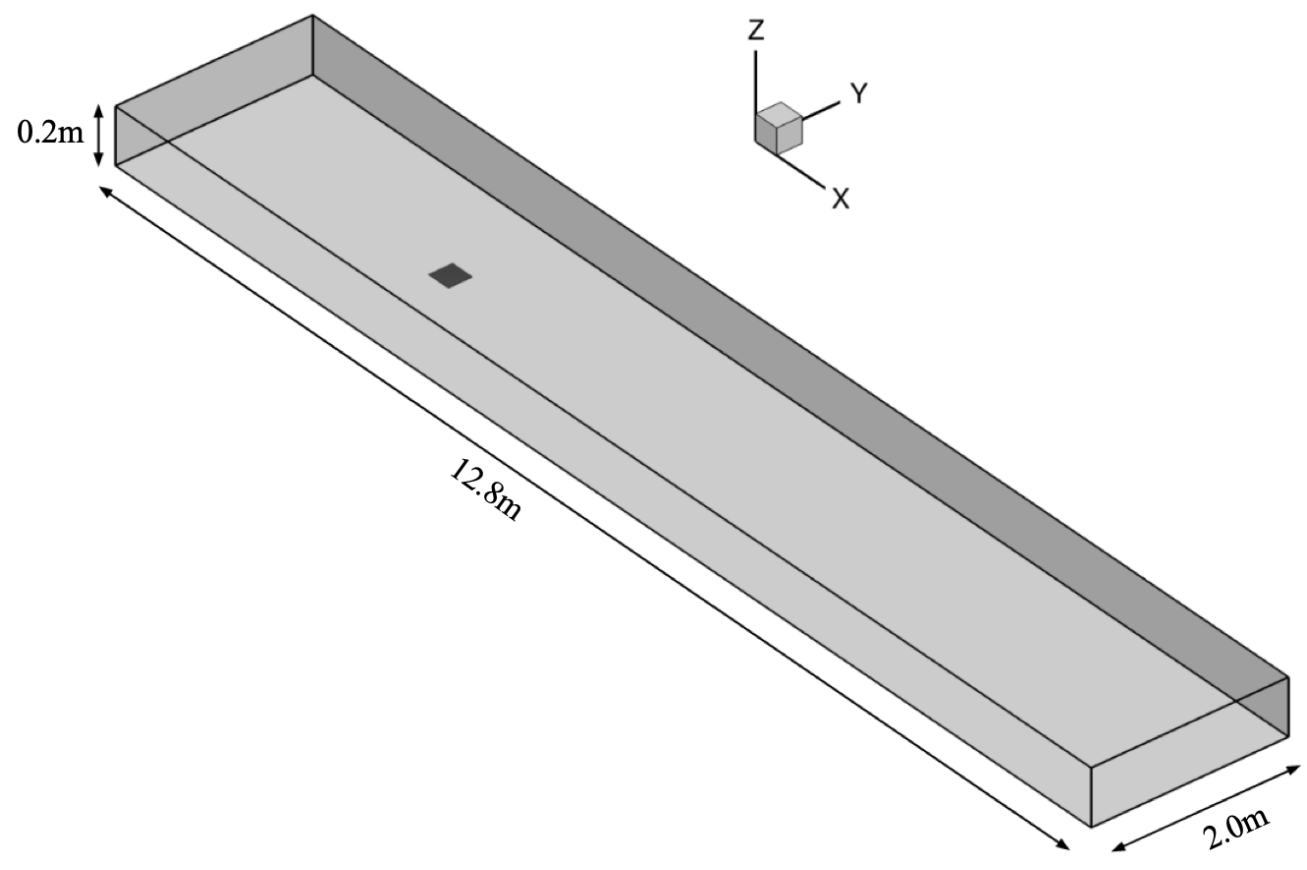

(a) 3D view 


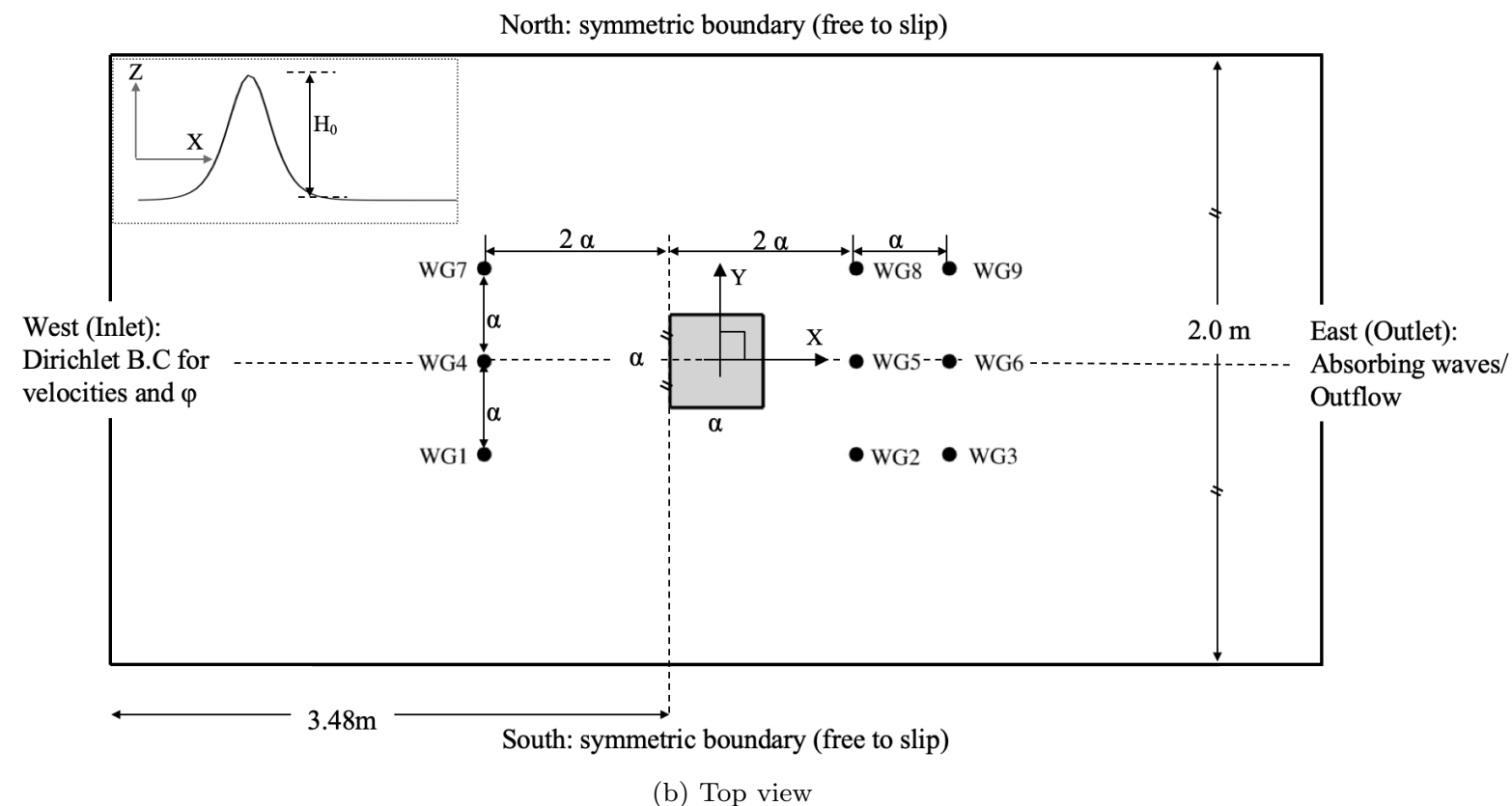

Fig. 8: Computational domain of the 3D wave-plate interaction case. (a) 3D view and (b) a top view schematic diagram of the computational set-up among with wave representation at the top-left corner

The grid resolution is the same as in the validation section, i.e. $\Delta x=0.0025 \mathrm{~m}, \Delta y=0.0025 \mathrm{~m}$, $\Delta z=0.00125 \mathrm{~m}(\mathrm{AR}=2)$ and $\Delta t=0.0005 \mathrm{~s}$, counting $5120 \times 800 \times 160$ computational cells. Dirichlet and Neumann boundary conditions are adopted for velocities and free-surface in the inlet and outlet boundaries, respectively. Neumann conditions for pressure are used in all boundaries and no-slip and free-slip walls are applied for the floor-bed and top wall, respectively. Here south and north walls act as symmetric boundaries (free to slip). Again IBM was used to apply the no-slip condition on the plate's surfaces. In order to consistently compare the current case with the infinitely wide plate, in the following referred to as '2D case', the $2 \mathrm{D}$ case has been re-simulated with the plate's LE located this time at $x=3.48 m(\sim 3 L)$ and wave-gauges WG1, WG2 and WG3 in Fig. 2 are relocated to agree with WG4, WG5, WG6 of the finite plate case, as shown in Fig. 8b. Due to relatively wide domain and fine uniform grid, simulations of the wave travelling over the 3D finite wide plate were carried out using 1024 CPU's and 168 hours of computational time.

\subsection{Results and discussion.}

\subsubsection{D finite square plate.}

Non-dimensional time series' of the water-surface and visualisation of local flow hydrodynamics are presented in this section to study the 3D wave-plate interaction. Six seconds of simulation time is recorded, enough for the wave to reach the plate, break and reflect as it travels further downstream. In the following figures, the $x$ - and $y$ - axes are normalised with the plate's length $\alpha$ and the $z$ - axis is normalised with the water-depth $d$. 

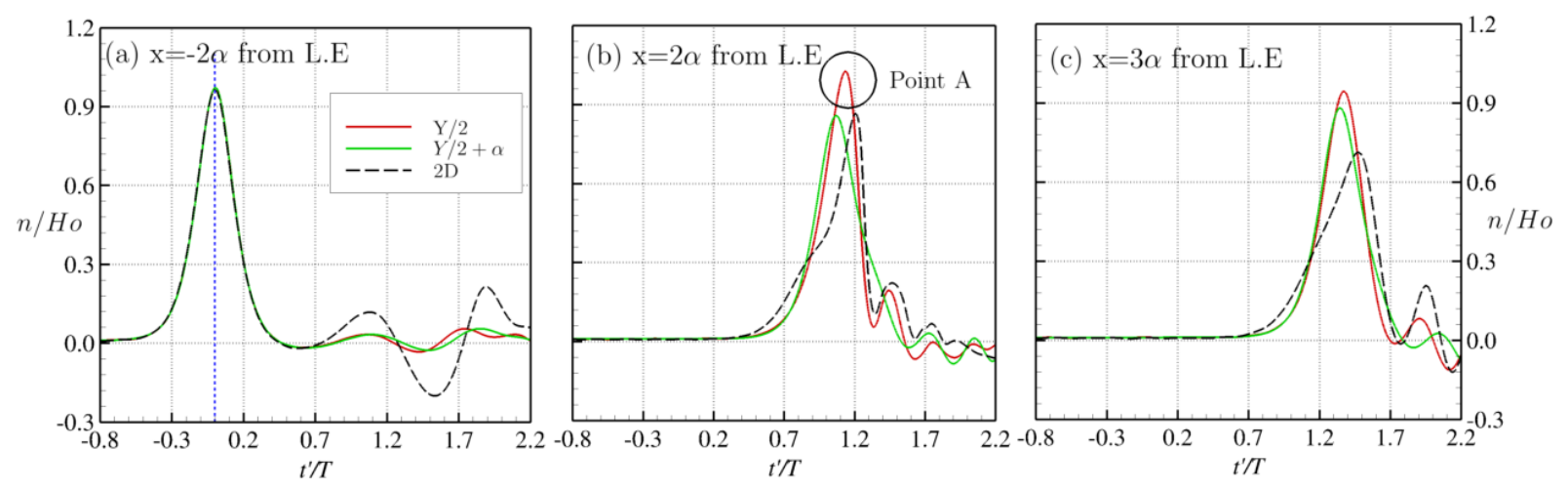

Fig. 9: Normalised wave elevations along the centreline $(Y / 2$, WG4-WG6) of the plate and at a lateral distance away from the centreline of the plate $(Y / 2+\alpha$, WG7-WG9) at (a) $x=-2 a$, (b) $x=2 a$ and (c) $x=3 a$ from the LE of the plate.

The non-dimensional wave elevations as a function of dimensionless time at various wave-gauges are plotted in Fig. 9. Due to the symmetrical shape of the plate, only the elevations recorded by WG4 to WG9 are presented, i.e elevations recorded by WG1 to WG3 at $Y / 2-\alpha$ are identical with those in WG7 to WG9 at $Y / 2+\alpha$, respectively. The dimensionless time $t^{\prime} / T=0$ is represented by the vertical dotted line in Fig. $9 \mathrm{a}$ and it is the instance at which the peak of the wave reaches the first wave-gauges (WG1,WG4 and WG7). Upstream of the plate the peaks of the $2 \mathrm{D}$ and $3 \mathrm{D}$ cases are identical, however wave reflections, starting at $t^{\prime} / T=0.7$ in Fig. 9a and $t^{\prime} / T=1.3$ in Fig. $9 \mathrm{~b}$ and $t^{\prime} / T=1.7$ in Fig. 9c, are quite different. Since the wave is free to refract in all directions reflected waves in the $3 \mathrm{D}$ case are generally lower than in the $2 \mathrm{D}$ case. Except for $2 \alpha$ downstream of the LE (Fig. 9b), where similar reflections to the 2D case are observed. This is mainly because WG5 is located near the breaking point, and the water surface primarily responds to the breaking wave. A higher peak in the wave elevation in the 3D case compared to the 2D case is observed, marked as Point A in Fig. 9b downstream of the finite plate and this feature is explained in more detail below.

Figure 10 visualises the wave propagation, steepening and breaking as it interacts with the plate in a 3D view as well as vortex structures around the plate using contours of the swirl strength at various instants in time. In this study swirl strength is calculated based on the imaginary part of the complex pair of eigenvalues of the velocity tensor according to Chong et al. [44] and Zhou et al. [45]. At Fig. 10a the wave approaches the plate and as it gets closer Fig. 10b a slight decrease of the free-surface above the plate (in relation to the still water level) is observed. Some fluid above the plate is already pushed downstream (due to the pressure gradient from the upstream wave) causing small clockwise-circulation eddy at the upper corner of the leading edge. When the wave travels over the plate, (Fig. 10c), the LE eddy gets more energetic and a counter clock-wise eddy starts to develop on the lower corner of the LE. Small eddies are formed at both corners of the plate's trailing edge (TE). What follows is a significant build-up of the wave as it propagates over the plate, which is due to the rapid decrease of the effective water-depth above the plate and the subsequent inrush of fluid from below the plate and the lateral sides where the water depth is greater (see 
Fig. 13b and Fig. 13c). Just before the wave breaks at Fig. 10e, vortices are shed behind the TE and a set of two opposite rotational eddies are captured. At the same time a strong coupled vortex below and above the LE is also observed. The wave breaks almost $0.5 \alpha$ downstream of the TE and reflections in all directions are recorded as the resulting wave propagates further away. As the waves propagates further downstream, leading and trailing edge eddies still oscillate and interact with the plate and water-surface at Fig. 10f. The shed eddies interact with the free-surface especially in such cases with shallow submergence depth $\left(d^{\prime} / d\right)$.

Smaller reflected waves are visible and appear to propagate in all directions.
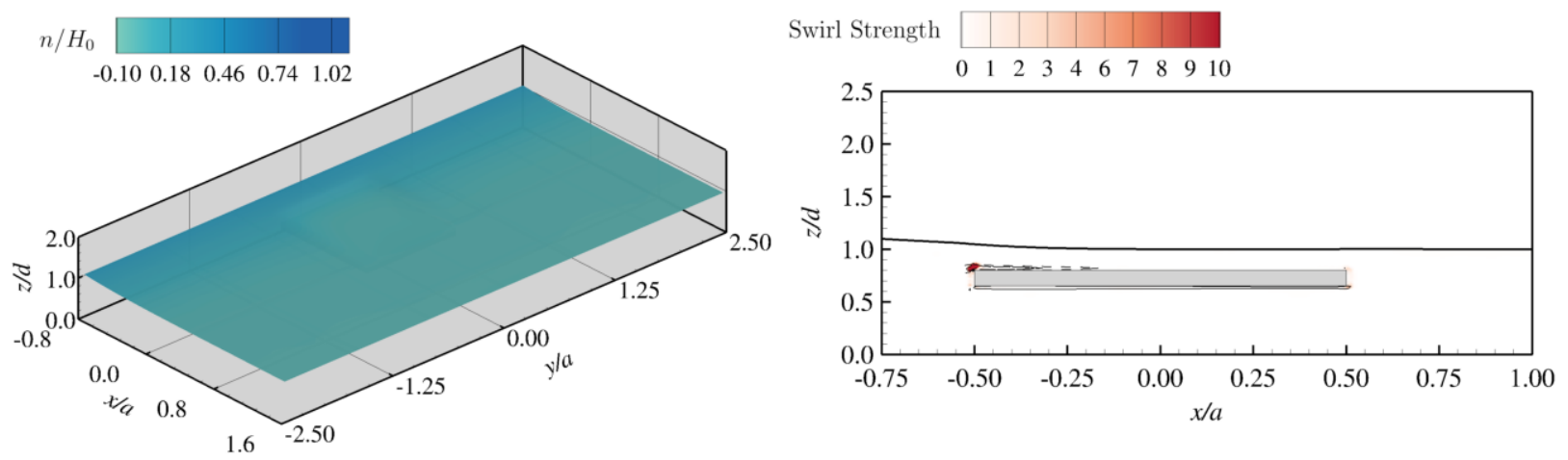

(a) $t^{\prime} / T=0.27$
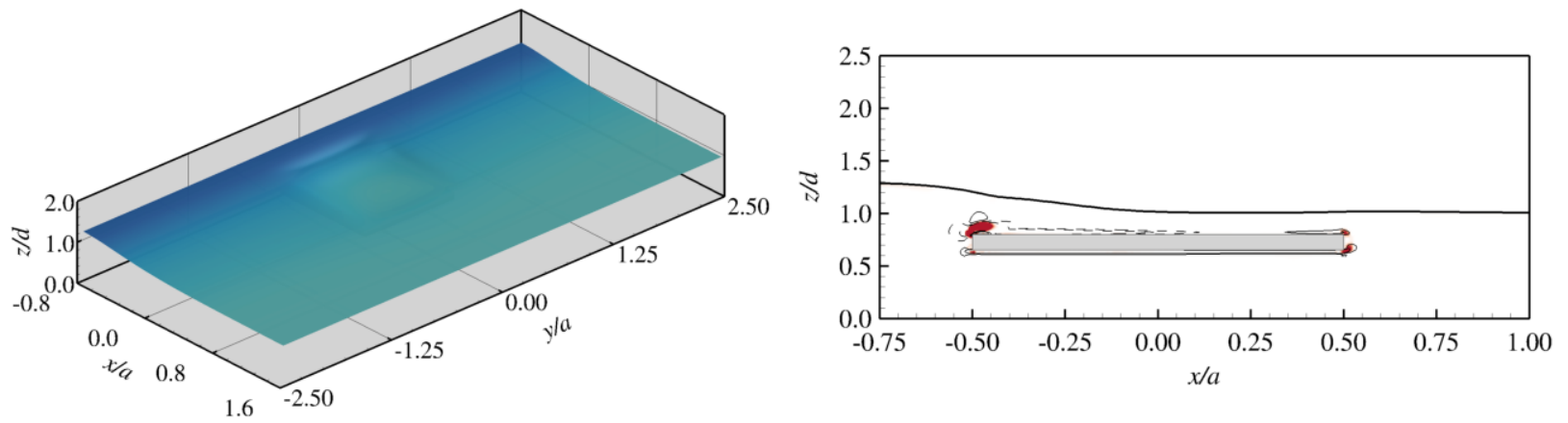

(b) $t^{\prime} / T=0.45$
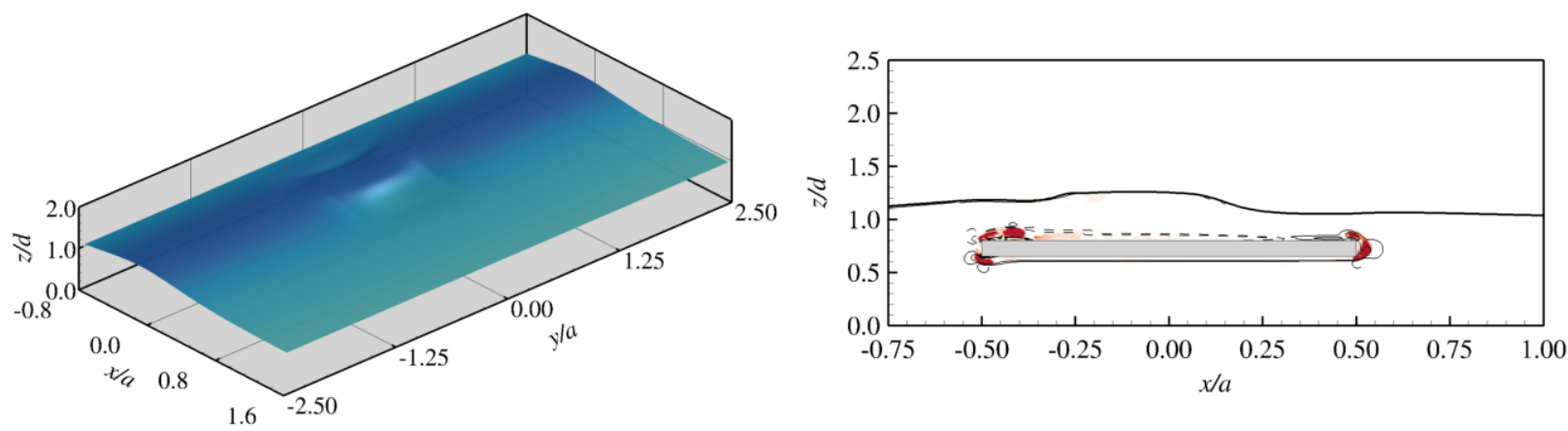

(c) $t^{\prime} / T=0.63$ 

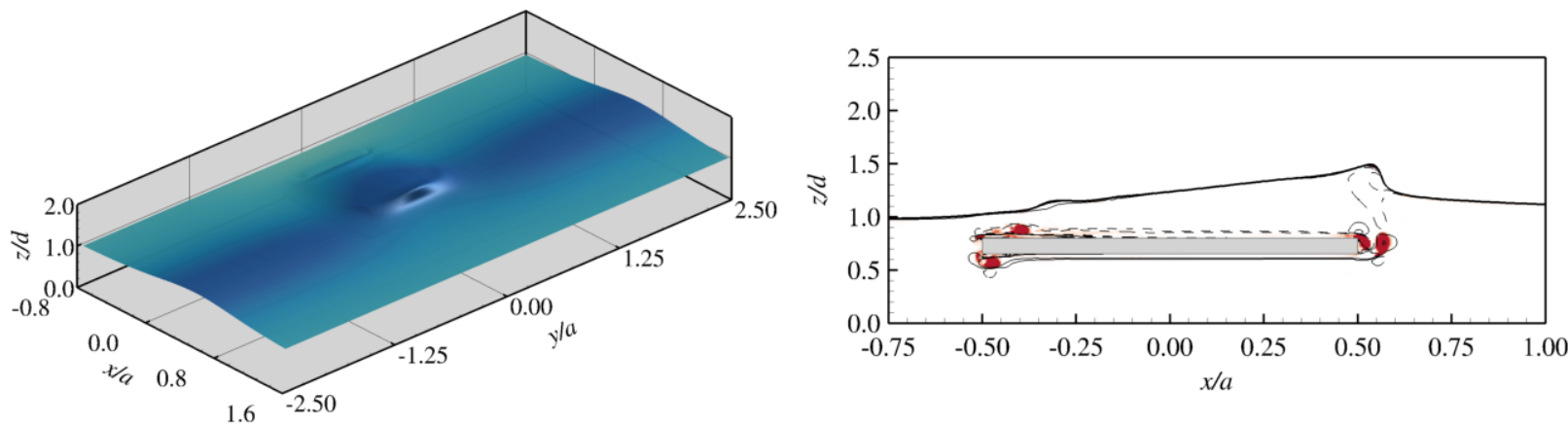

(d) $t^{\prime} / T=0.81$
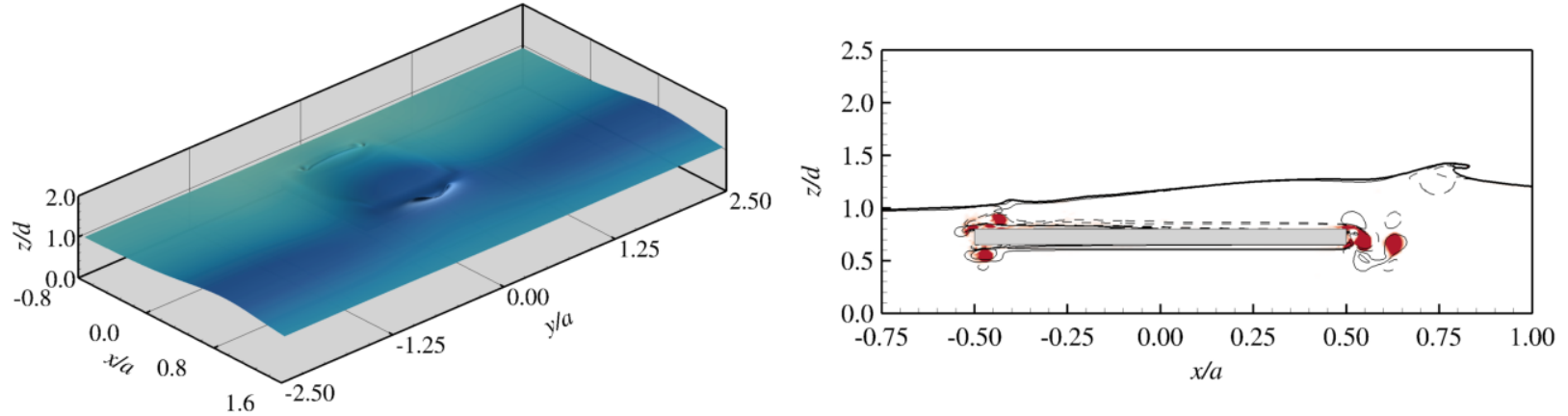

(e) $t^{\prime} / T=0.90$
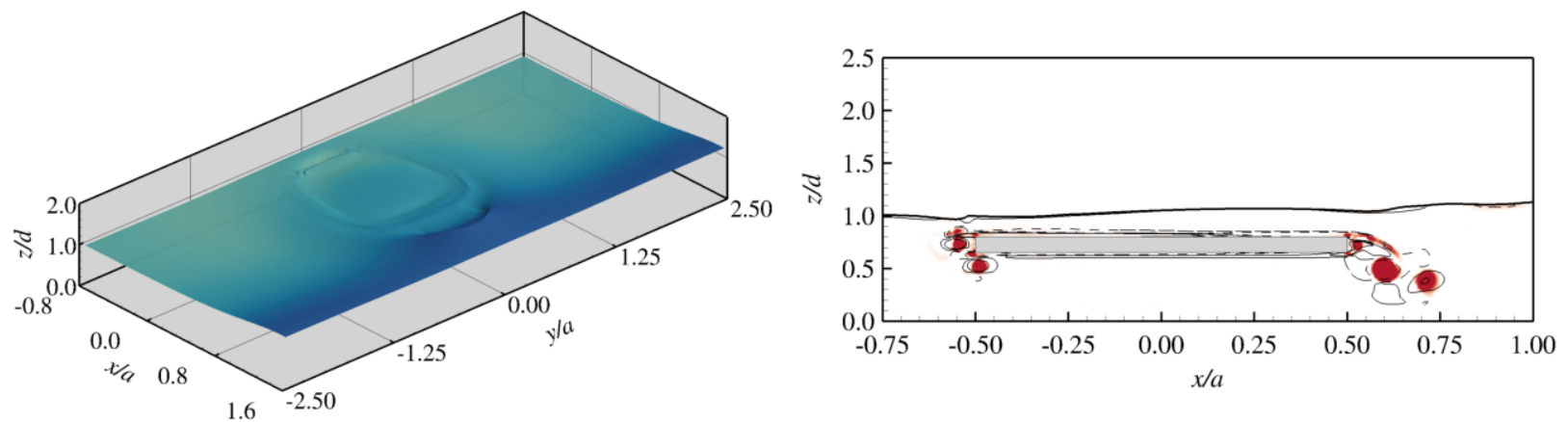

(f) $t^{\prime} / T=1.08$

Fig. 10: Wave Propagation and breaking. Left: Iso-surface of $\phi=0$, Right: Contour of swirl strength and lines of y-vorticty (dashed lines=negative vorticity). Sliced at $y / a=0$.

Figure 11 presents contours of the normalized pressure in the vicinity of the plate at two instants in time. The hydrostatic pressure has been removed and the remaining dynamic pressure is normalised with the pressure head $P_{0}=\rho g H_{0}$. A pressure difference between the lower and upper side of the plate is obvious before and after the wave has reached the structure, Figs. 11a and 11b respectively. A similar phenomenon was also observed and explained in Lo and Liu [2] in a relevant experimental study. The initial pressure 
difference is developed due to the immediate response of the flow below the plate when almost zero velocity above is recorded as shown in Fig. 12 and hence higher pressure below the plate is observed, Fig. 11a. The almost uniform flow below the plate, again presented in Fig. 12 is driven by the pressure difference between the two stream-wise openings of the plate. This initial higher pressure below the plate also explains the early uplift force recorded in Fig. 5 followed by a downward force due to the weight of the incident wave in Fig. 11b.

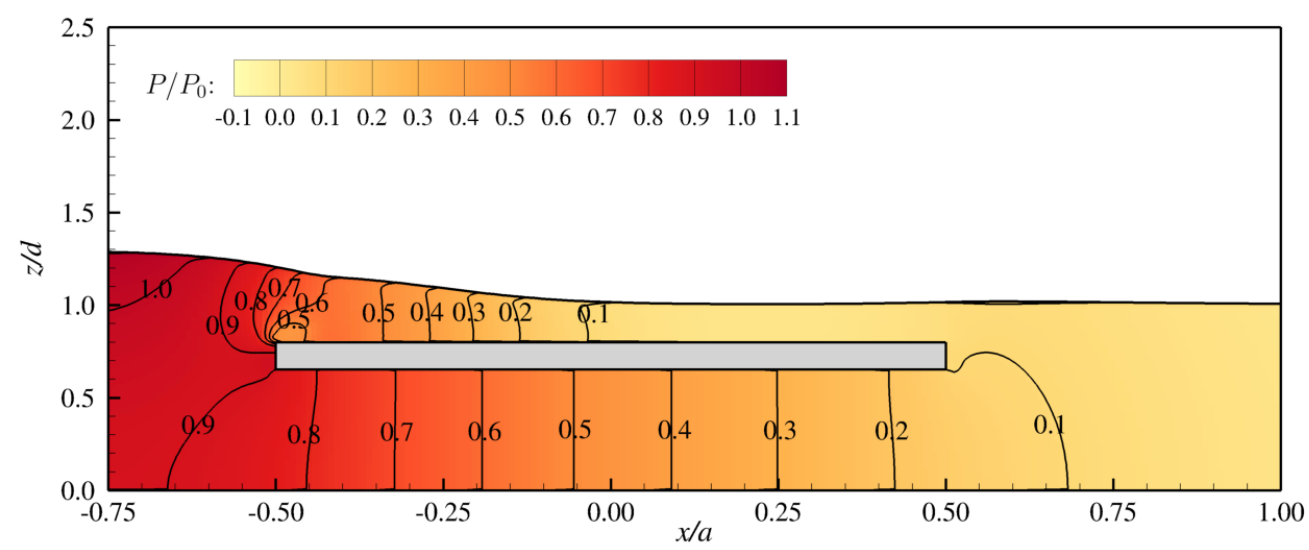

(a) $t^{\prime} / T=0.45$

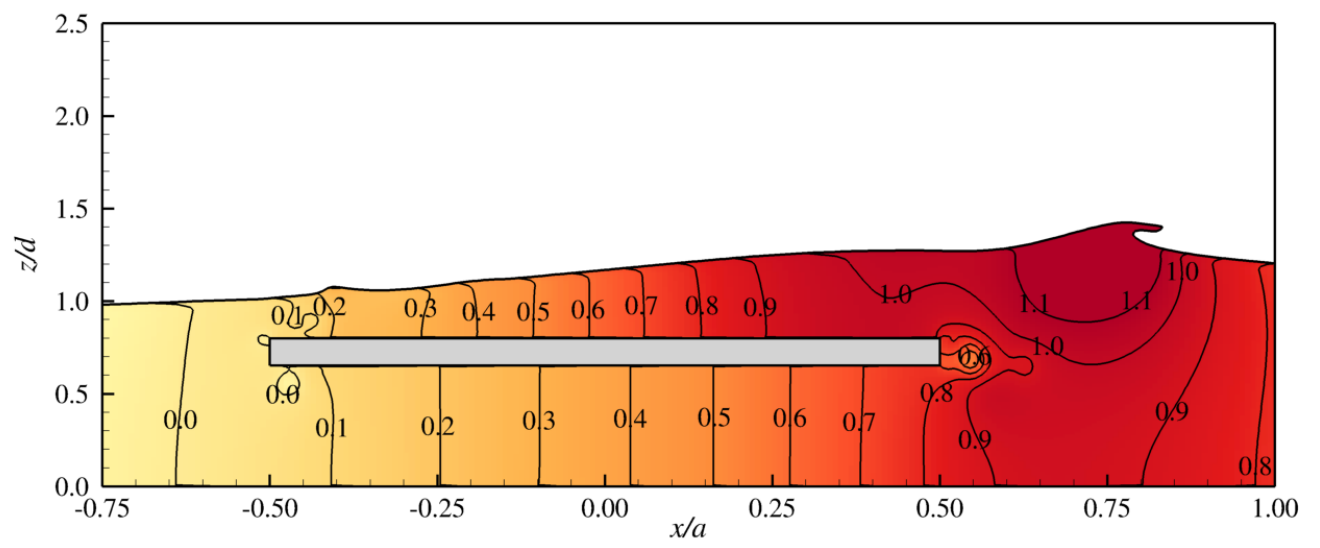

(b) $t^{\prime} / T=0.90$

Fig. 11: Contours of the normalized pressure near the plate. Sliced at $y / a=0$.

A span-wise flow, demonstrated in Fig. 13, is observed while the wave interacts with the submerged plate. The span-wise flow can be explained as follows: When the wave is close to the plate, the higher pressure below and lower pressure above the plate drives a span-wise flow from the lower to the upper side of the plate. As a result, two clock-wise eddies are generated at the plate's corners, Figs. 13b and 13c. In addition, fluid enters the region above the plate due to traveling from the sides of the plate towards the upper surface 
due to the low pressure. The "additional mass" generates a higher wave elevation above the plate than in the $2 \mathrm{D}$ case. Once the wave has passed the plate, a secondary span-wise flow, this time from the region above the plate to the region below the plate is observed. As a result, corner vortices are generated again but this time these are rotating in the opposite direction (Figs. 13e and 13f).

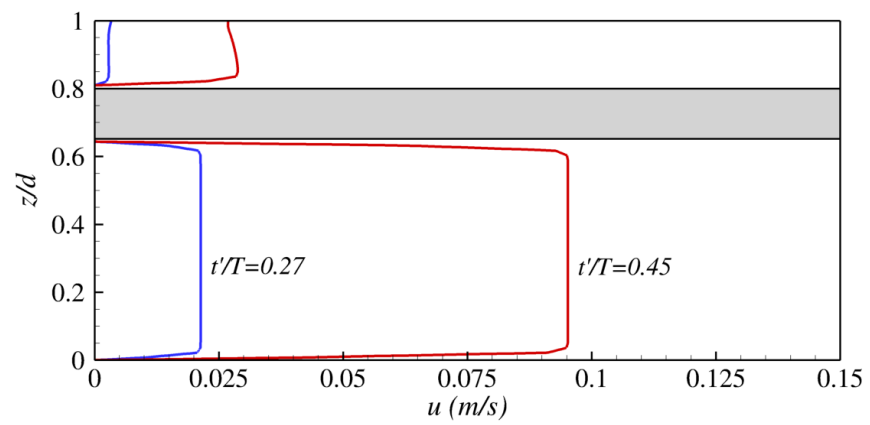

Fig. 12: Profile of the horizontal velocity $u=f(z)$ at $x / a=y / a=0$.

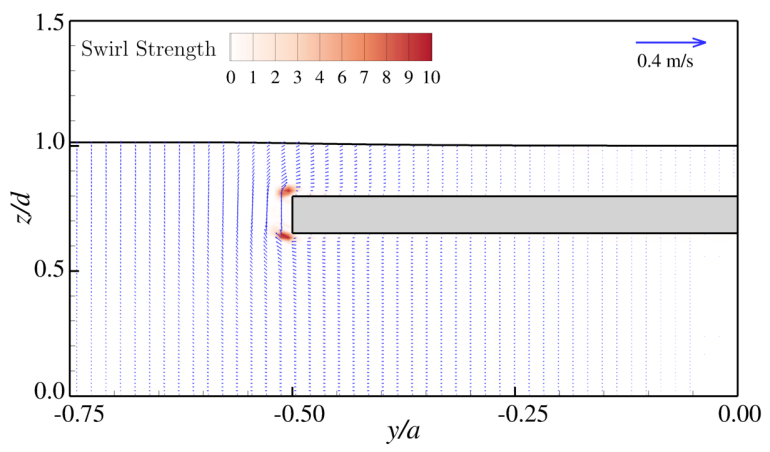

(a) $t^{\prime} / T=0.27$

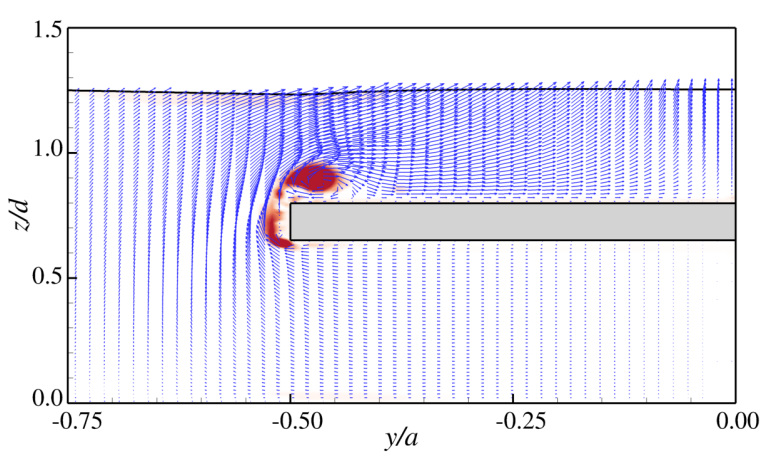

(c) $t^{\prime} / T=0.63$

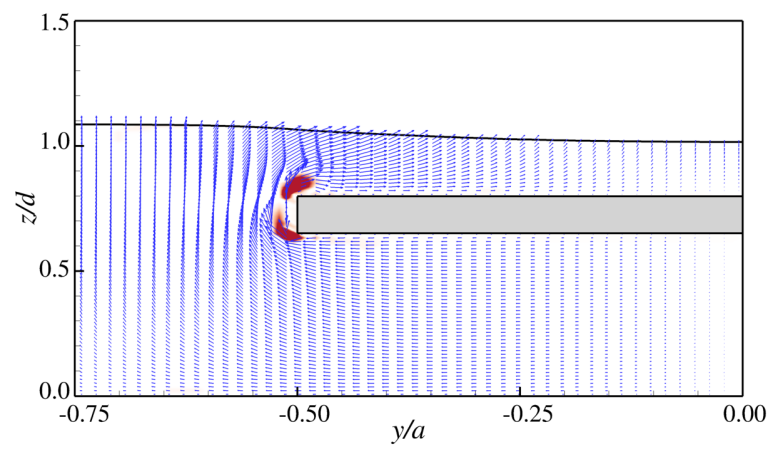

(b) $t^{\prime} / T=0.45$

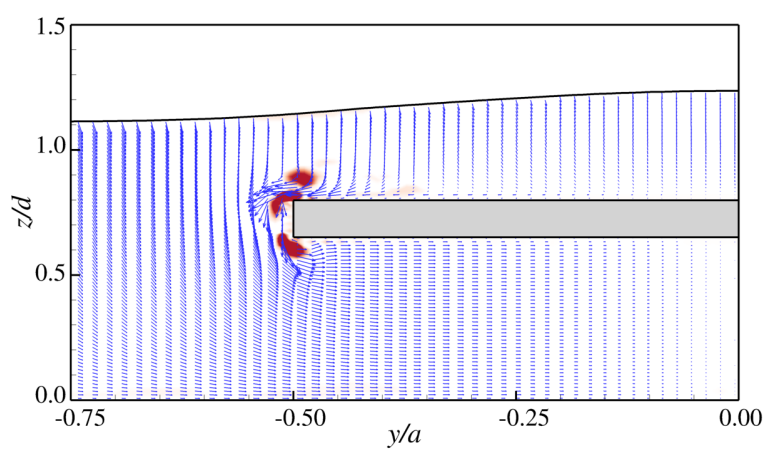

(d) $t^{\prime} / T=0.81$ 


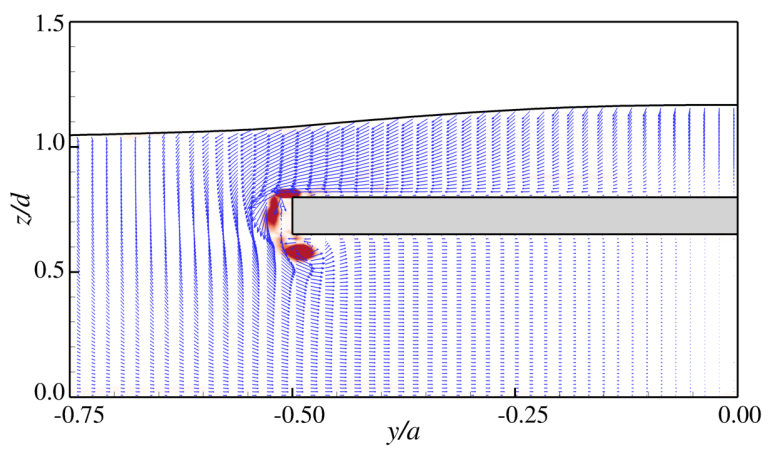

(e) $t^{\prime} / T=0.90$

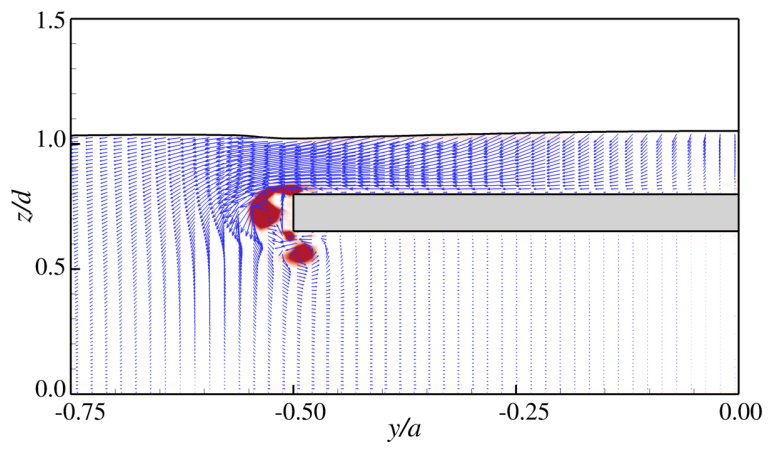

(f) $t^{\prime} / T=1.08$

Fig. 13: Contour of the swirl strength and velocity vectors. Sliced at $x / a=0$. Vector plotted every second cell.

Figure 14 compares the simulated free-surfaces of the finite plate and infinitely wide case, just before wave-breaking occurs. The wave elevation of the finite plate corresponds to the $x-z$ plane sliced over the centre-line of the plate. Figure 14 confirms the variations between the two cases and the existence of three-dimensional effects. When a finite plate is under consideration, a higher wave compared to the 2D case exists, also captured by WG5 and marked as point A in Fig. 9b, due to the 'additional mass' brought by the initial span-wise flow described above. In addition to that, the breaking point of the square-like plate is located further downstream in relation to the $2 \mathrm{D}$ case. This is mainly, due to the flow acceleration as the wave passes over the plate and the higher wave celerity due to larger wave height, $c=\sqrt{g\left(H_{0}+d\right)}$. Due to that, a time-shift in the wave elevation between the finite and infinitely wide plate is also observed in Fig. 9b and Fig. 9c.

Figure 15 presents the calculated vertical force $\bar{F}_{z}$ per unit width (a) and the horizontal force $\bar{F}_{x}$ per unit width per unit thickness (b), obtained from the finite plate simulation (solid lines) and the infinite plate simulation (dashed lines), respectively. The differences in $\bar{F}_{z}$ are due to three-dimensional effects, i.e. in the form of the spanwise flow from below the finite plate to the upper side of the plate (see Figs. 13a and 13b) in response to the pressure difference above and below the plate, whereas in the $2 \mathrm{D}$ case this flow is prohibited. Additionally, the larger wave above the 3D plate adds extra weight (downward force) and hence decreases the vertical force (uplift) on the plate. Quantitatively, the peak uplift of the 3D plate is approximately $40 \%$ less than the peak uplift of the 2D plate. Similarly, a decrease of about $30 \%$ in the downward force, is computed for the 3D case, a result of the span-wise flow (see Figs. 13d to 13f) which increases the pressure below the plate opposing the weight of the wave propagating above. Furthermore, three-dimensional effects reduce the 'active' period of time of forces acting on the plate, due the higher wave celerity over the 3D plate. The horizontal forces (Fig. 15b) are hardly affected by the geometry of the plate, which is due to plate being so thin. 


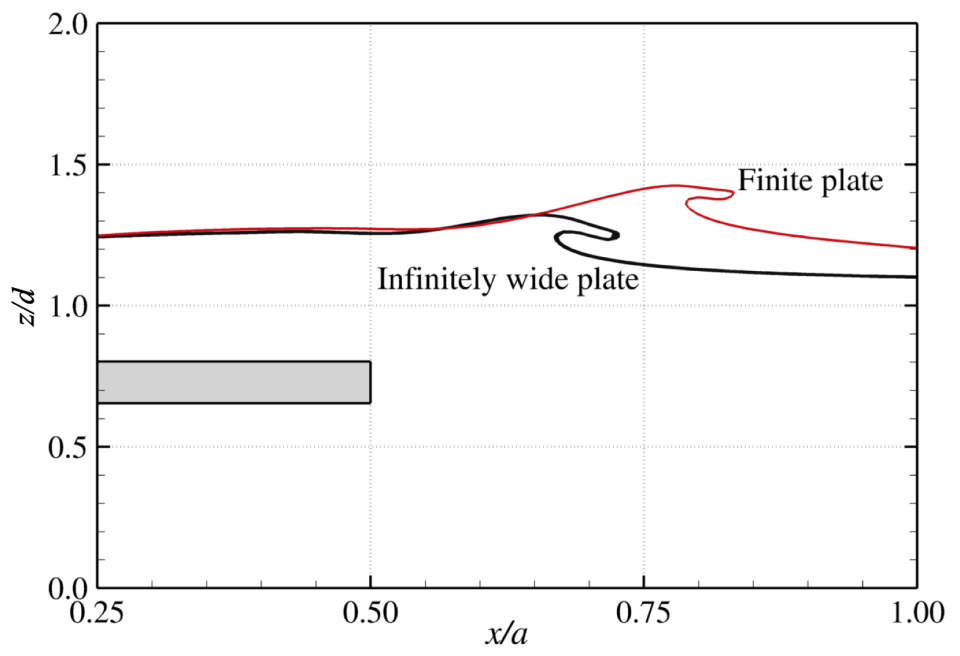

Fig. 14: Calculated water-surface elevation near the plate, just before wave-breaking occurs. Finite plate (solid red line) sliced at $y / a=0$ and infinitely wide plate (solid black line).
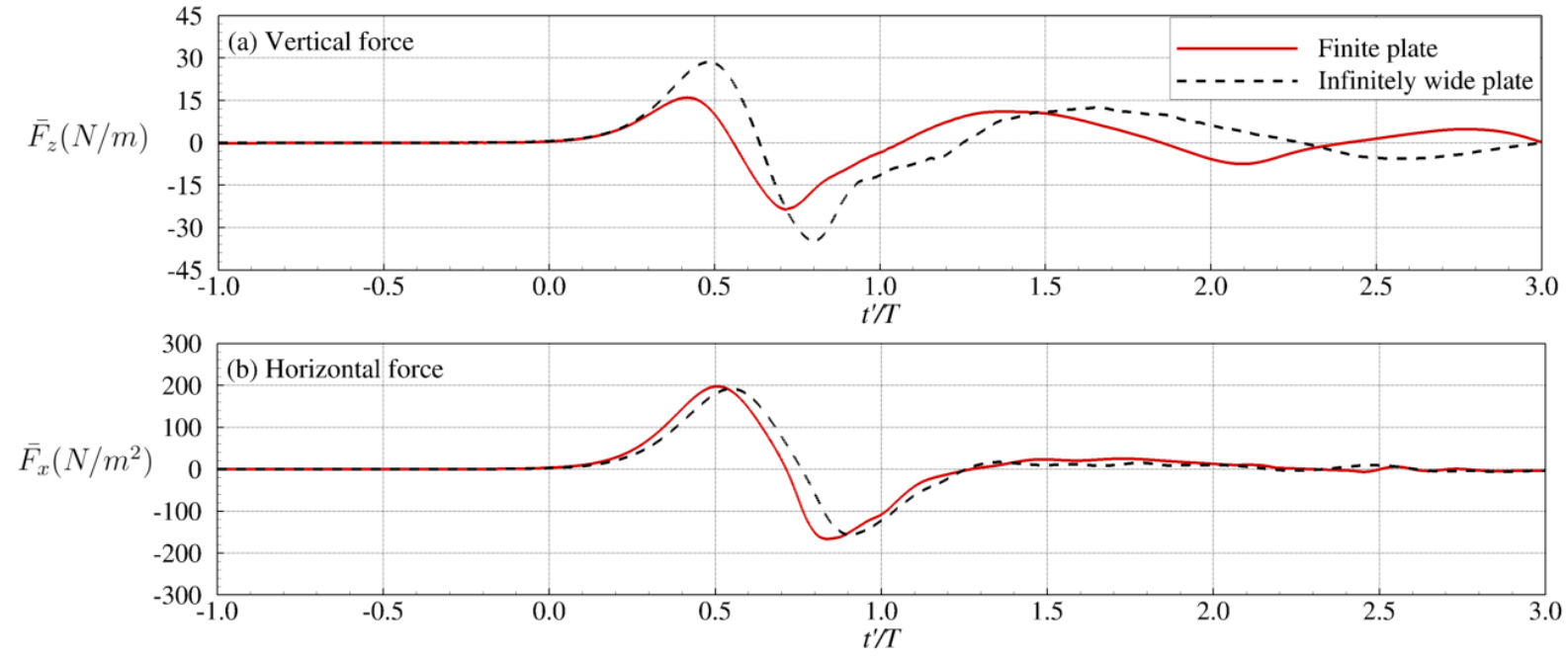

Fig. 15: Comparisons of the simulated (a) vertical force per unit width, $\bar{F}_{z}$ and (b) horizontal force per unit width per unit thickness, $\bar{F}_{x}$ for the finite plate (solid lines) and infinitely wide (dashed lines) fixed plate.

A three-dimensional plot of an isosurface of the Q-criterion $(Q=100)$ coloured with the horizontal velocity is presented in Fig. 16. It visualises aforementioned eddies near the plate, both stream-wise and span-wise eddies. The eddies at the lateral edges of the plate are significantly smaller than the ones near the leading and trailing edges due to the dominance of the stream-wise flow. An interesting feature is the occurrence of vortices at the corners of the plate, best observed from the downstream looking upstream, Fig. 16b, revealing the symmetry of the flow and associated vortex structure. 

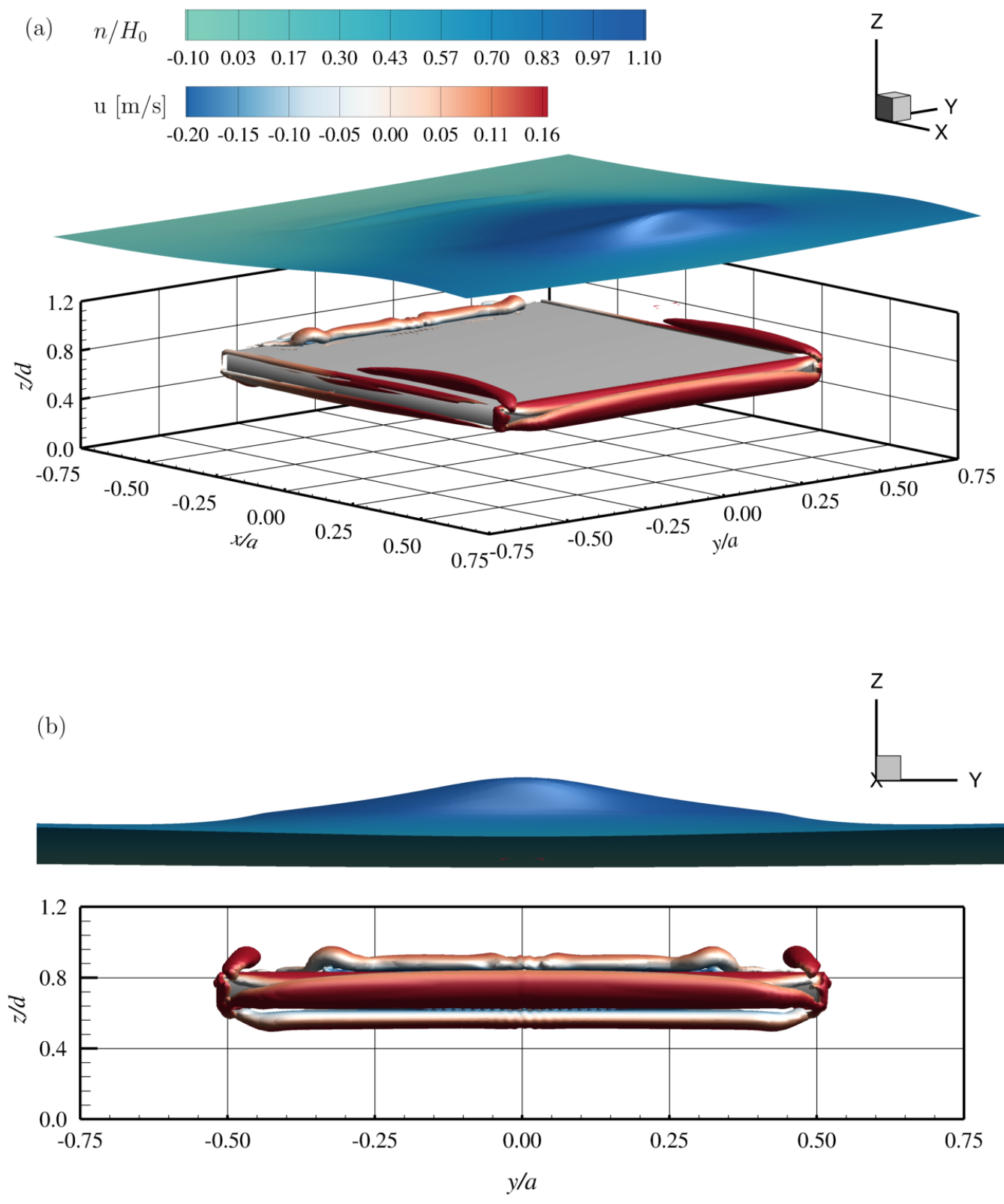

Fig. 16: (a) 3D projection and (b) view from TE of the iso-surface of $\phi=0$ and Q-criterion= 100 coloured by horizontal velocity $u$ at $t^{\prime} / T=0.81$. Iso-surface of $\phi=0$ is shifted upwards for better visualization.

\subsubsection{Effect of angle of attack.}

In this section, three-dimensional effects of the wave-plate interaction are investigated further by extending the previous simulations to a solitary wave interacting with a submerged rotated plate. In real offshore environments, waves approach a structure from different directions. Thus, quantification of the influence 
of wave direction on wave propagation and acting hydrodynamic forces is required to derive generally valid for which the wave travels a maximum distance over the plate in the streamwise direction. The wave-plate interaction is expected to yield complex flow structures that may affect the integral behaviour of the plate. Figure 17 describes the problem showing the rotated plate (solid red line) and the corresponding angle of attack $\theta_{i}$.

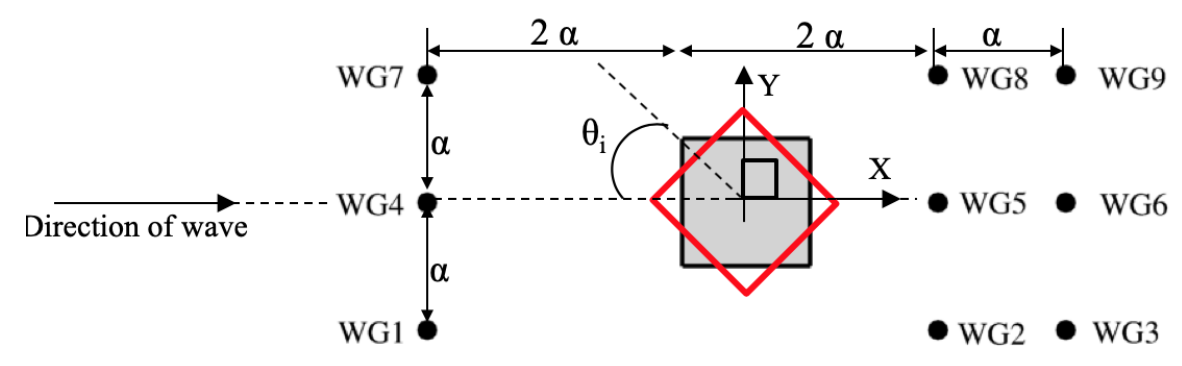

Fig. 17: Schematic diagram of the plate rotated at an angle $\theta=45^{\circ}$ (red line) and $\theta=0^{\circ}$ (black line).

The same computational set-up as before Section 4.1 and Fig. 8 is adopted and $\theta_{i}=45^{\circ}$ is chosen to compute and further understand the complex hydrodynamics and three dimensional effects of a wave traveling over a rotated finite plate as well as on the forces acting on the plate.

Figure 18 visualises the occurrence of large eddies using the Q-criterion, coloured with the horizontal velocity $u$, as well as the evolution of the free-surface as the wave propagates over the rotated plate. The the plate $\left(d^{\prime} / d=0.2\right)$ and the large effective diameter of the eddies, larger than $0.2 z / d$ at certain times, fluctuations on the free-surface are built and shaped according to the eddies' pattern. Vortices are generated due to the primary streamwise and secondary spanwise flow evolution discussed in section 4.2 .1 and plotted in $x-z$ and $y-z$ planes in Fig. 10 right column and Fig. 13, respectively. As the wave approaches the plate's LE at Fig. 18a two large clockwise eddies roll-up near the two upstream edges on the upper side of the plate, as a result of the dominant streamwise flow, which are then detached and propagate over the structure, at Fig. 18b, reflecting in terms of fluctuations on the free-surface. Similarly, eddies are formed at the downstream edges at both lateral sides of the plate and grow as the wave builds-up at Fig. 18c. After wave breaking, (Fig. 18d), most of the vortices have detached from the structure while upstream (LE) eddies 

(marked with point $\mathrm{P})$.

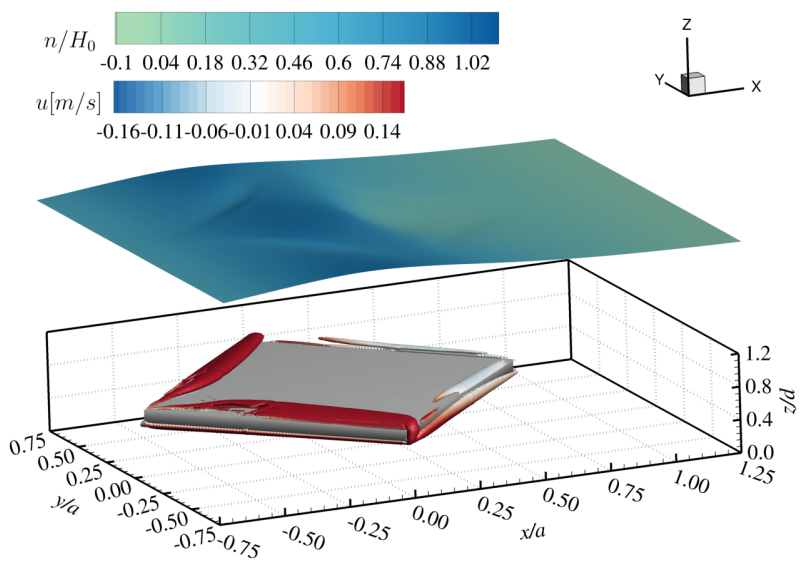

(a) $t^{\prime} / T=0.63$

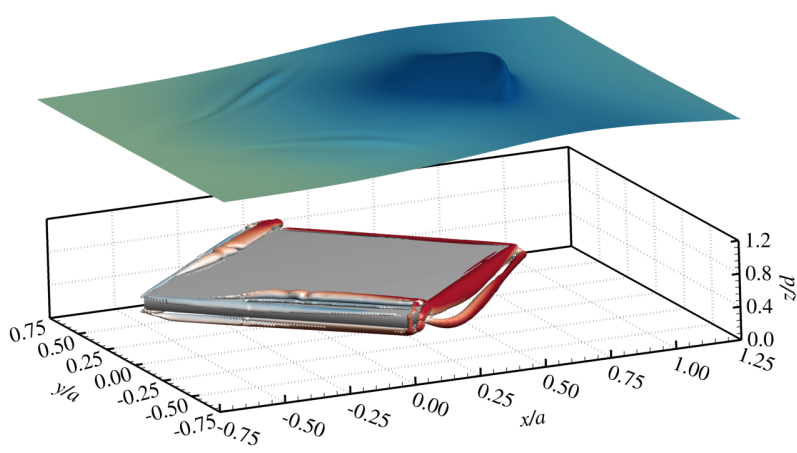

(c) $t^{\prime} / T=0.85$

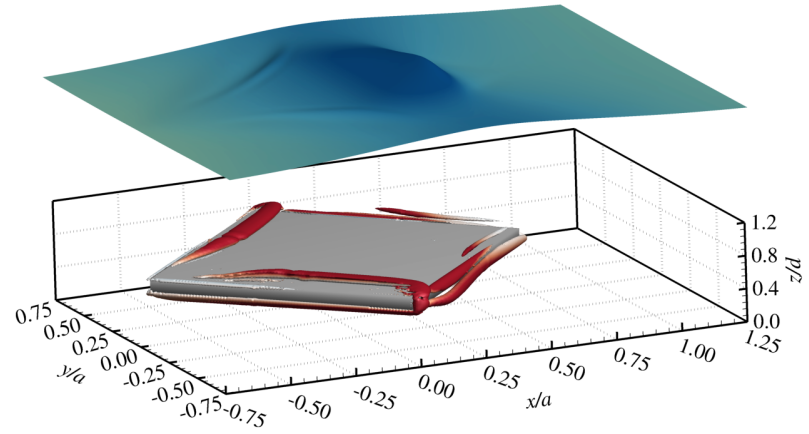

(b) $t^{\prime} / T=0.74$

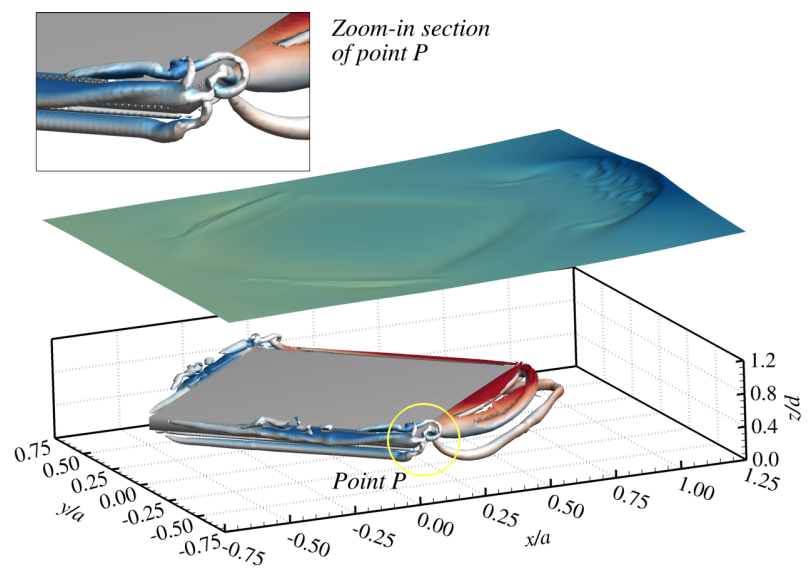

(d) $t^{\prime} / T=1.08$

Fig. 18: Screenshots of the iso-surface of Q-criterion=100 coloured by horizontal velocity $\mathrm{u}$ and iso-surface of $\phi=0$ coloured by normalized elevation $n / H_{0}$ at several time-steps. Iso-surface of $\phi=0$ is shifted upwards for better visualization.

Figure 19a plots the water surface over the rotated plate, moments before wave breaking occurs, together with the corresponding non-rotated $\left(\theta_{i}=0^{\circ}\right)$ and infinitely wide $(2 \mathrm{D})$ plates, sliced at $y / a=0$. As discussed before, the interaction of the wave with the two non-rotated plates (3D and 2D) generates similar water surfaces, however, differences in the wave elevation are observed when the plate is rotated at $45^{\circ}$. As a result of the larger streamwise distance between the leading and trailing edge of the rotated plate at $y / a=0$, the wave includes a forward jet and hence breaks at a further downstream location compared to the other two cases. Figure 19b compares the free-surface of the two finite plates in terms of the distance from the TE, $x^{*} / \alpha$, where $x^{*} / \alpha=0$ at plate's TE. From this, the characteristic distance from the TE, between the two 
$3 \mathrm{D}$ cases before breaking occurs is almost the same where the non-rotated plate features a more dominant forward jet at the peak of the wave. In addition to that, a shallower mean water-level above the structure is observed when a $\theta_{i}=45^{\circ}$ plate is considered but in both $3 \mathrm{D}$ cases the wave rises up to the same vertical location before breaking.

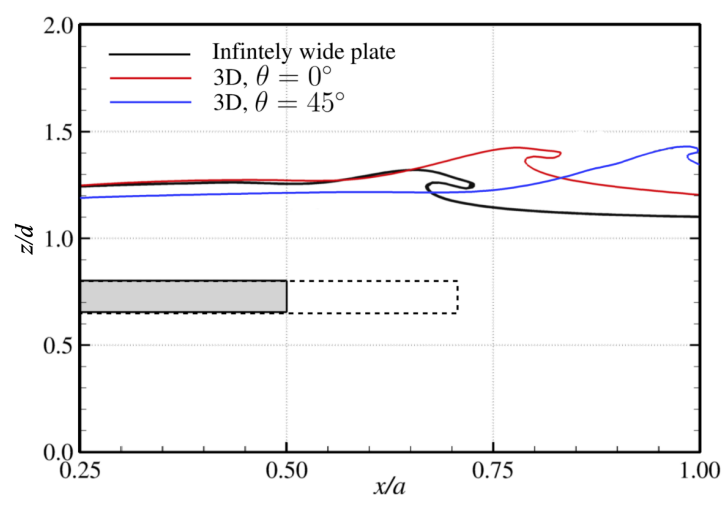

(a)

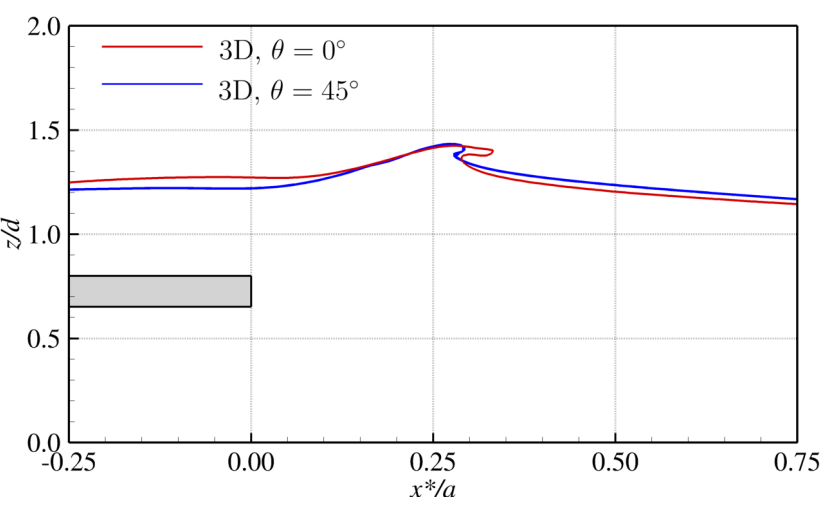

(b)

Fig. 19: Calculated water-surface elevation near the plate. $\theta_{i}=0^{\circ}$ (solid red line), $\theta_{i}=45^{\circ}$ (solid blue line) sliced at $y / a=0$ and infinitely wide plate (solid black line). In (a) dashed-line presents the cross-section of the $\theta_{i}=45^{\circ}$ plate and in (b) x-axis represents the distance from the $\mathrm{TE} x^{*} / \alpha$.

Figure 20 shows the time-history of the wave elevation recorded at the nine wave-gauges (see also Fig. $8 \mathrm{~b}$ ). The water surface variations are almost identical in both cases apart from WG5. This is due to a very similar wave progression, i.e wave's build-up, plunging jet and breaking, found in both cases. The main differences in wave elevation is the breaking location and minor fluctuations observed over the edges of the plate. The latter appear as a result of the lower eddies which are either stationary or propagating forward and hence not presented during the time-period the wave-gauges record. Due to the breaking location in $\theta_{i}=45^{\circ}$ case (i.e closer to WG5 but further upstream, Fig. 18d) a higher peak and unique tail fluctuations are captured by WG5 in Fig. 20e. Beyond that, the propagation, interaction and reflection of the incident solitary wave is almost similar in both three-dimensional finite plates. This is mainly due to the relatively long wave compared to the plate's length $(L / \alpha \approx 4)$ and hence the angle of attack does not strongly affect the wave elevation. 

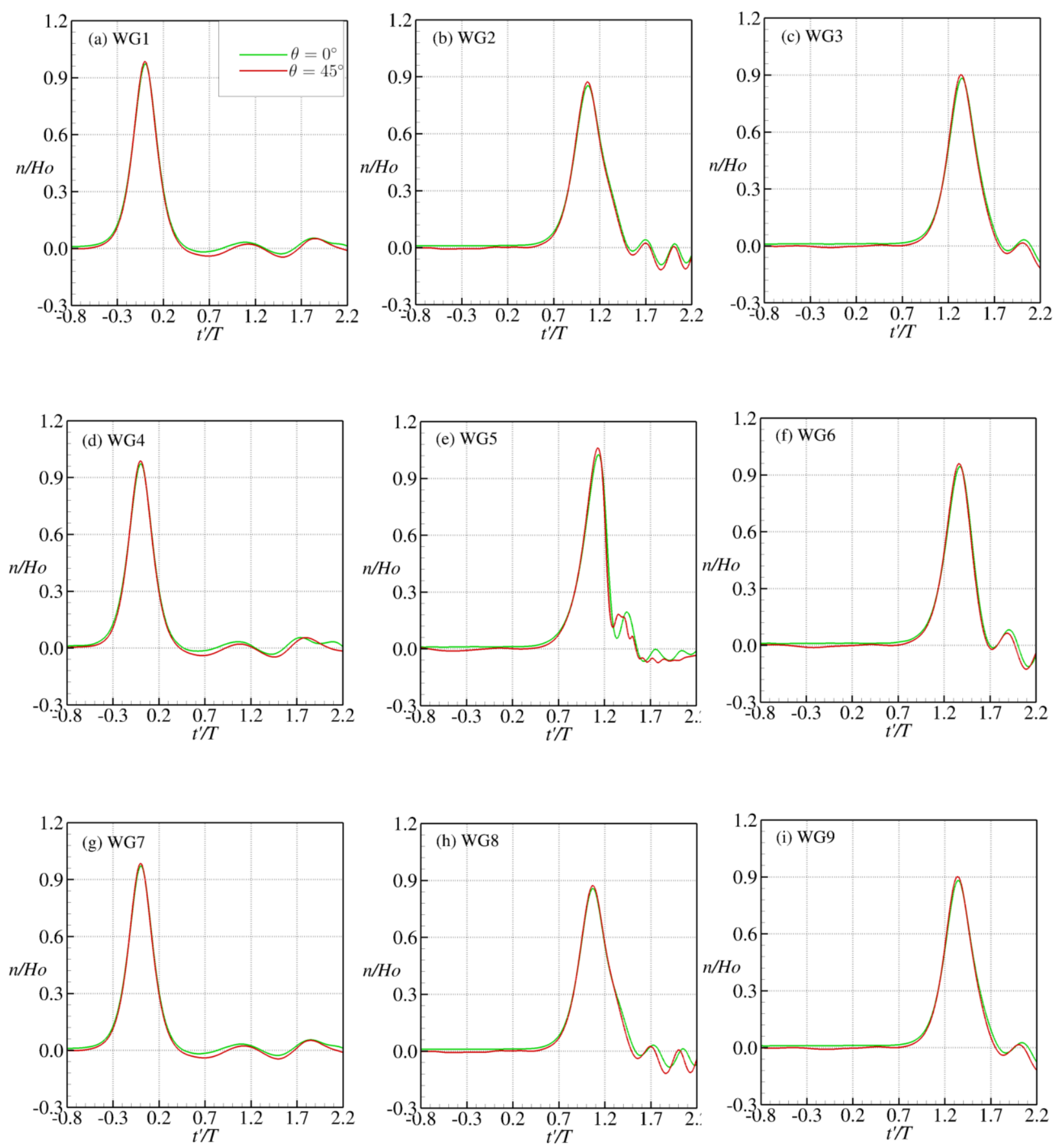

Fig. 20: Normalised wave elevations recorded by WG1-WG9. $\theta_{i}=0^{\circ}$ (solid green lines) and $\theta_{i}=45^{\circ}$ (solid red lines).

Figure 21 compares the vertical force per unit width $\bar{F}_{z}$ and horizontal force per unit width per unit thickness $\bar{F}_{x}$ acting on the 2D and 3D $\theta_{i}=0^{\circ}$ and $\theta_{i}=45^{\circ}$ plates. Since the variations between the $2 \mathrm{D}$ and $3 \mathrm{D}$ cases were discussed in the previous section we now focus on the differences between the two 3D cases. It is clear that the direction of the wave affects the hydrodynamic forces acting on the structure. In the case of a solitary wave approaching the plate from exactly $45^{\circ}$ degrees, both vertical and horizontal forces are 


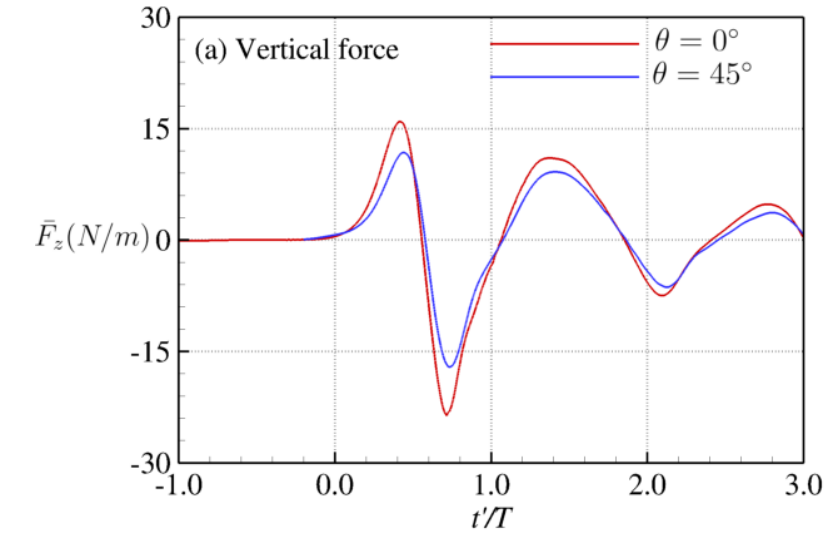

reduced. In Fig. 21a the peak uplift force calculated in the $\theta_{i}=45^{\circ}$ case has been quantitatively reduced by $26 \%$ compared to the corresponding $\theta_{i}=0^{\circ}$ uplift force. Similarly, downward peak force has been reduced by a further $27.5 \%$ resulting in a total $66 \%$ and $57.5 \%$ decrease in uplift and downward force, respectively compared to the 2D case. As already mentioned, the secondary spanwise flow presented only in the 3D cases is responsible for the force reduction. The further decrease in vertical force acting on the rotated plate may be due to the shorter distance the flow must travel from the lower side of the plate to the upper side (initial uplift force) and from the upper to the lower side (downward force). Moreover, the secondary uplift and downward forces, from $t^{\prime} / T=1.2$ and forward, are also decreased however by a lesser degree.

The same phenomenon is observed in the horizontal force (Fig. 21b) in which the positive peak has been decreased by $25 \%$ and the negative peak by $14 \%$. The reduction in horizontal peak forces is mostly expected due to the shape of the rotated plate. As the wave approaches, the streamwise flow interacts with the front face of the structure resulting to high pressure and thus a positive horizontal force. In the case of the $\theta_{i}=0^{\circ}$ plate the front face is perpendicular to the direction of the wave resulting to higher pressures compared to the sloped front face of the $\theta_{i}=45^{\circ}$ plate. Furthermore, the initial streamwise flow has a greater effect on the structure than the secondary backward flow, occurring when the wave builds-up and suction occurs (Fig. 18c), and hence the reduction in the positive peak is greater.

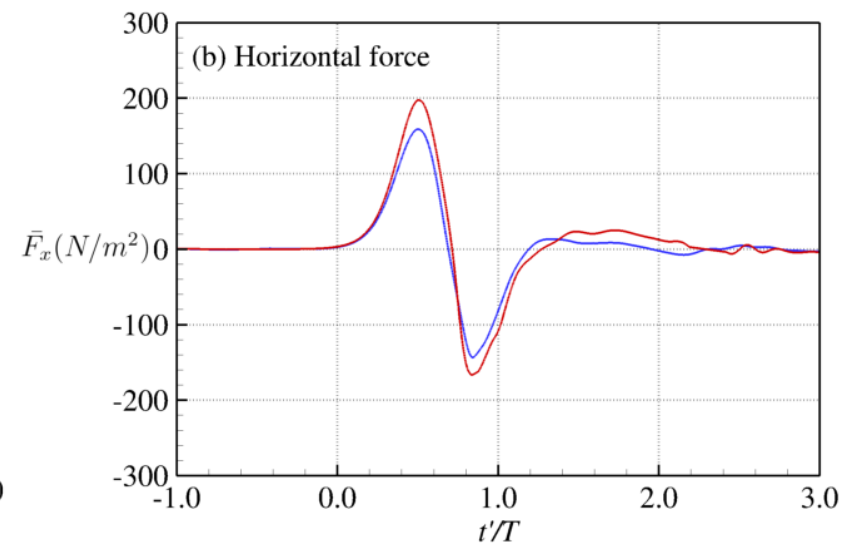

Fig. 21: Comparisons of the simulated (a) vertical force per unit width, $\bar{F}_{z}$ and (b) horizontal force per unit width per unit thickness, $\bar{F}_{x}$ for the $\theta_{i}=0^{\circ}$ (solid red lines), $\theta_{i}=45^{\circ}$ (solid blue lines) finite plates.

\section{Conclusions}

In this paper results of numerical simulations of solitary-wave-plate interaction using the in-house code Hydro3D have been presented and discussed. The code was validated first using experimental data and results of previous Euler model (no viscous effects) simulations for solitary wave propagation over an infinitely wide plate. Overall, convincing agreement in wave elevation has been observed. It can also be concluded that for this particular case, Hydro3D produced slightly more accurate free-surface elevations in comparison with the (inviscid) Euler model, demonstrating that viscous effects are non-negligible. A parametric validation 
study of horizontal and vertical forces on an infinitely wide submerged plate yielded convincing agreement between numerical and experimental data. Vertical forces increase with steeper waves and lower plate

submergence albeit submergence is found to have little effect on horizontal forces. Successful validation of the code allowed studying the effect of finiteness of the submerged plate. Significant differences in the flow and wave elevations in comparison with the infinite plate have been revealed demonstrating the presence of three-dimensional effects. Streamwise and spanwise energetic eddies near the edges of the plate have been observed and visualised. These are the result of lateral and vertical pressure gradients. The former is the result of a depression of the water surface above the plate as the wave approaches the plate whereas the latter is the result of different streamwise velocities above and below the plate. The spanwise flow results in little separation eddies at the lateral edges of the plate. Stronger eddies occur at the corners of the leading and trailing edges of the plate, a result of streamwise flow separation. Another remarkable outcome of the current study is the significant decrease in the peaks of uplift and downward force, quantified to be $40 \%$ and $30 \%$ less respectively, due to the spanwise flow and three-dimensional effects, in case of the finite plate. The exerted horizontal force, however, remained unchanged when finiteness effects have been examined. Finally the effect of angle of attack on wave-plate interaction has been examined. Forces are affected by the direction of the incident wave. Peak lift and drag forces acting on the rotated plate are reduced by approximately $20 \%$ in comparison to the original (zero degree) finite plate, whereas water levels around the plate are very similar. Plate finiteness and direction of the incident wave (or plate rotation) does not affect negatively the design and cost of submerged structures.

\section{References}

[1] Hayatdavoodi, M., Ertekin, R.C.. Review of wave loads on coastal bridge decks. Applied Mechanics Reviews 2016;68(3). doi:10.1115/1.4033705.

[2] Lo, H.Y., Liu, P.L.. Solitary waves incident on a submerged horizontal plate. Journal of Waterway, Port, Coastal and Ocean Engineering 2014;140(3):1-17. doi:10.1061/(ASCE)WW.1943-5460.0000236.

[3] Seiffert, B., Hayatdavoodi, M., Ertekin, R.C.. Experiments and computations of solitary-wave forces on a coastal-bridge deck. Part I: Flat Plate. Coastal Engineering 2014;88:194-209. doi:10.1016/j . coastaleng. 2014.01.005.

[4] Hayatdavoodi, M., Ertekin, R.C.. Wave forces on a submerged horizontal plate - Part I: Theory and modelling. Journal of Fluids and Structures 2015;54:566-579. doi:10.1016/j.jfluidstructs. 2014. 12.010.

[5] Hayatdavoodi, M., Ertekin, R.C.. Wave forces on a submerged horizontal plate-Part II: Solitary and cnoidal waves. Journal of Fluids and Structures 2015;54:580-596. doi:10.1016/j · jfluidstructs . 2014.12.009. 
[6] Ai, C., Ma, Y., Yuan, C., Dong, G.. Semi-implicit non-hydrostatic model for 2D nonlinear wave interaction with a floating/suspended structure. European Journal of Mechanics, B/Fluids 2018;72:545560. doi:10.1016/j. euromechflu.2018.08.003.

[7] Xie, Z., Yan, S., Ma, Q., Stoesser, T., Lu, L.. Three-dimensional numerical study of solitary waves interacting with a horizontal plate. In: International Ocean and Polar Engineering Conference. 2019, p. 2733-2738.

[8] Yu, X.. Functional Performance of a Submerged and Essentially Horizontal Plate for Offshore Wave Control: A Review. Coastal Engineering Journal 2002;44(2):127-147. doi:10.1142/ S0578563402000470.

[9] Siew, P.F., Hurley, D.G.. Long surface waves incident on a submerged horizontal plate. Journal of Fluid Mechanics 1977;83(1):141-151. doi:10.1017/S0022112077001098.

[10] Patarapanich, M., Cheong, H.F.. Reflection and transmission characteristics of regular and random waves from a submerged horizontal plate. Coastal Engineering 1989;13(2):161-182. doi:10.1016/ 0378-3839 (89) 90022-7.

[11] Liu, P.L.F., Iskandarani, M.. Scattering of short-wave groups by submerged horizontal plate. Journal of Waterway, Port, Coastal and Ocean Engineering 1991;117(3):235-246. doi:10.1061/(ASCE) 0733-950X (1991) 117:3(235).

[12] Yu, X., Chwang, A.T.. Analysis of wave scattering by submerged circular disk. Journal of Engineering Mechanics 1993;119(9):1804-1817. doi:10.1061/(ASCE) 0733-9399(1993) 119:9(1804).

[13] Brossard, J., Chagdali, M.. Experimental investigation of the harmonic generation by waves over a submerged plate. Coastal Engineering 2001;42(4):277-290. doi:10.1016/S0378-3839(00) 00064-8.

[14] Rey, V., Touboul, J.. Forces and moment on a horizontal plate due to regular and irregular waves in the presence of current. Applied Ocean Research 2011;33(2):88-99. doi:10.1016/j .apor. 2011.02.002.

[15] Poupardin, A., Perret, G., Pinon, G., Bourneton, N., Rivoalen, E., Brossard, J.. Vortex kinematic around a submerged plate under water waves. Part I: Experimental analysis. European Journal of Mechanics, B/Fluids 2012;34:47-55. doi:10.1016/j.euromechflu. 2012.02.003.

[16] Liu, C., Huang, Z., Keat Tan, S.. Nonlinear scattering of non-breaking waves by a submerged horizontal plate: Experiments and simulations. Ocean Engineering 2009;36(17-18):1332-1345. doi:10 . 1016/j. oceaneng.2009.09.001.

[17] Brossard, J., Perret, G., Blonce, L., Diedhiou, A.. Higher harmonics induced by a submerged horizontal plate and a submerged rectangular step in a wave flume. Coastal Engineering 2009;56(1):1122. doi:10.1016/j.coastaleng.2008.06.002. 
[18] Gotoh, H., Okayasu, A., Watanabe, Y.. Computational Wave Dynamics; vol. 37 of Advanced Series on Ocean Engineering. World Scientific; 2013. ISBN 978-981-4449-70-0. doi:10.1142/8714.

[31] Uhlmann, M.. An immersed boundary method with direct forcing for the simulation of particulate flows. Journal of Computational Physics 2005;209(2):448-476. doi:10.1016/j · jcp. 2005.03.017. 
[32] Kara, M., Stoesser, T., McSherry, R.. Calculation of fluid structure interaction: methods, refinements, applications. Proceedings of the Institution of Civil Engineers - Engineering and Computational Mechanics 2015;168(2):59-78. doi:10.1680/eacm.15.00010.

[33] Ouro, P., Stoesser, T.. An immersed boundary-based large-eddy simulation approach to predict the performance of vertical axis tidal turbines. Computers and Fluids 2017;152:74-87. doi:10.1016/j . compfluid.2017.04.003.

[34] Ouro, P., Fraga, B., Lopez-Novoa, U., Stoesser, T.. Scalability of an Eulerian-Lagrangian large-eddy simulation solver with hybrid MPI/OpenMP parallelisation. Computers and Fluids 2019;179:123-136. doi:10.1016/j.compfluid.2018.10.013.

[35] Kara, S., Stoesser, T., Sturm, T.W., Mulahasan, S.. Flow dynamics through a submerged bridge opening with overtopping. Journal of Hydraulic Research 2015;53(2):186-195. doi:10.1080/00221686 . 2014.967821.

[36] Kara, S., Kara, M.C., Stoesser, T., Sturm, T.W.. Free-surface versus rigid-lid LES computations for bridge-abutment flow. Journal of Hydraulic Engineering 2015;141(9):1-9. doi:10.1061/(ASCE) HY . $1943-7900.0001028$.

[37] McSherry, R., Chua, K., Stoesser, T., Mulahasan, S.. Free surface flow over square bars at intermediate relative submergence. Journal of Hydraulic Research 2018;56(6):825-843.

[38] Osher, S., Sethian, J.A.. Fronts propagating with curvature-dependent speed: Algorithms based on Hamilton-Jacobi formulations. Journal of Computational Physics 1988;79(1):12-49. doi:10.1016/ 0021-9991 (88) 90002-2.

[39] Shu, C.W.. High Order Weighted Essentially Nonoscillatory Schemes for Convection Dominated Problems. SIAM Review 2009;51(1):82-126. doi:10.1137/070679065.

[40] Sussman, M.. A level set approach for computing solutions to incompressible two-phase flow. Journal of Computational Physics 1994;114(1):146-159. doi:10.1006/jcph.1994.1155.

[41] Lee, J.J., Skjelbreia, J.E., Raichlen, F.. Measurement of velocities in solitary waves. Journal of the Waterway, Port, Coastal and Ocean Division 1982;108(WW2):200-218. doi:10.1061/(ASCE) 0733-950X (1983) 109:3(353).

[42] Choi, J., Yoon, S.B.. Numerical simulations using momentum source wave-maker applied to RANS equation model. Coastal Engineering 2009;56(10):1043-1060. doi:10.1016/j.coastaleng.2009.06. 009.

[43] Perić, R., Abdel-Maksoud, M.. Reliable damping of free-surface waves in numerical simulations. Ship Technology Research 2016;63(1):1-13. doi:10.1080/09377255.2015.1119921. 
[44] Chong, M.S., Perry, A.E., Cantwell, B.J.. A general classification of three-dimensional flow fields. Physics of Fluids A 1990;2(5):765-777. doi:10.1063/1.857730.

[45] Zhou, J., Adrian, R.J., Balachandar, S., Kendall, T.M.. Mechanisms for generating coherent packets of hairpin vortices in channel flow. Journal of Fluid Mechanics 1999;387:353-396. doi:10.1017/ S002211209900467X. 